\title{
Fuzzy data envelopment analysis in the presence of undesirable outputs with ideal points
}

\author{
Ali Ebrahimnejad ${ }^{1}$ (i) $\cdot$ Naser Amani $^{2}$
}

Received: 4 July 2020 / Accepted: 28 September 2020 / Published online: 27 October 2020

(c) The Author(s) 2020

\begin{abstract}
Data envelopment analysis (DEA) is a prominent technique for evaluating relative efficiency of a set of entities called decision making units (DMUs) with homogeneous structures. In order to implement a comprehensive assessment, undesirable factors should be included in the efficiency analysis. The present study endeavors to propose a novel approach for solving DEA model in the presence of undesirable outputs in which all input/output data are represented by triangular fuzzy numbers. To this end, two virtual fuzzy DMUs called fuzzy ideal DMU (FIDMU) and fuzzy anti-ideal DMU (FADMU) are introduced into proposed fuzzy DEA framework. Then, a lexicographic approach is used to find the best and the worst fuzzy efficiencies of FIDMU and FADMU, respectively. Moreover, the resulting fuzzy efficiencies are used to measure the best and worst fuzzy relative efficiencies of DMUs to construct a fuzzy relative closeness index. To address the overall assessment, a new approach is proposed for ranking fuzzy relative closeness indexes based on which the DMUs are ranked. The developed framework greatly reduces the complexity of computation compared with commonly used existing methods in the literature. To validate the proposed methodology and proposed ranking method, a numerical example is illustrated and compared the results with an existing approach.
\end{abstract}

Keywords Data envelopment analysis · Fuzzy relative efficiency · Undesirable outputs · Ideal and anti-ideal units

\section{Introduction}

Performance evaluation is a critically important procedure for the companies and organizations operating in the modern business world, where survival in the fiercely competitive business environment requires maintaining high levels of performance and efficiency. Thus, performance evaluations of business and production units in its many aspects have long been of interest to organizational managers and corporate executives. One of the major challenges ahead of proper performance evaluation is the lack of access to an accurate production function because of the complexity of the production process, the change in production technology, the impact of external factors on performance, the large size of data and operations, and the abrupt changes in policy

Ali Ebrahimnejad

a.ebrahimnejad@qaemiau.ac.ir; aemarzoun@gmail.com

1 Department of Mathematics, Qaemshahr Branch, Islamic Azad University, Qaemshahr, Iran

2 Department of Applied Mathematics, University of Texas at Austin, Austin, USA to address acute problems. Access to production function is of immense importance for microeconomic analyses, as it allows managers to easily evaluate the performance of business units. Parametric methods have long been among the leading methods of estimation of production function and thereby unit performance. In these methods, user assumes a particular form for production function and then determines the parameters of the function with the help of mathematical techniques [7].

Farrel [22] proposed a non-parametric method for this application, which later became the basis of another method called data envelopment analysis (DEA). DEA was developed by Charnes et al. [11] to solve unit performance evaluation problems with the Constant Returns-to-Scale (CRS). DEA is a non-parametric programming technique for measuring the relative efficiency of multiple decisionmaking units (DMUs) tasked with transforming multiple inputs into multiple outputs. In DEA, the weights are set in such a way as to maximize the performance of the evaluated unit (DMU) relative to the rest of the units. To evaluate the performance of DMUs, one must first determine the production possibility set (PPS) and then obtain the projection of

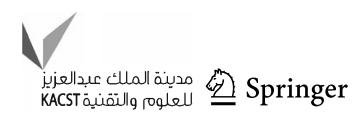


DMUs on the efficient boundary (frontier) of PPS. This frontier represents the maximum output that can be obtained by a particular input, or the least input that is needed to produce a particular output. The DMUs that are located on this frontier are called efficient and the remaining DMUs are inefficient. Any inefficient DMU can be made efficient by reducing its inputs or increasing its outputs. Banker et al. [8] expanded the DEA theory for the Variable Returns-to-Scale (VRS). In DEA, the efficiency level is very sensitive to the initial data, so the use of scattered data will lead to significant variations in DMUs' efficiency levels. Furthermore, in the theoretical problems and especially those commonly solved by DEA models, inputs and outputs are usually expressed with exact values. This is often inconsistent with the reality of business world, where, in many cases, our knowledge about the production process is inaccurate and the available data are imprecise or obscure. This deficiency has been the primary driver for the combination of DEA models with fuzzy set theory. The general assumption of DMU performance evaluation is that efficiency can be improved by reducing inputs or increasing outputs. The traditional models like CCR and $\mathrm{BCC}$ are based on this assumption. But it is false to assume that all organizations seek to minimize all their inputs and maximize all their outputs, as inputs and outputs could be desirable or undesirable based on the process and objectives. Therefore, such cases require a different analytical approach that could account for desirability and undesirability of parameters.

The main contributions of this paper are sevenfold: (1) a novel DEA framework in crisp and fuzzy environments is developed to overall assessing of each DMU in the presence of undesirable outputs. (2) In contrast to most existing approaches, which provide crisp efficiency measures, the developed fuzzy framework provides fuzzy efficiency measures. (3) In contrast to existing method [26], two virtual fuzzy DMUs called fuzzy ideal DMU (FIDMU) and fuzzy anti-ideal DMU (FADMU) are introduced into fuzzy DEA (FDEA) in the presence of undesirable outputs. (4) In contrast to existing method $[26,50]$, the exact form of membership function of the fuzzy relative closeness is determined to give an overall assessment of each DMU. (5) According to the developed framework, because of using fuzzy arithmetic approach, less linear programming models are solved in order to full ranking of DMUs in comparison with Puri and Yadav's method [50] and Hatamai-Marbini et al.'s method [26]. (6) In contrast to existing method [50] that evaluates the performance of each fuzzy DMU based on optimistic perspective, the proposed framework evaluates each fuzzy DMU from both the optimistic and pessimistic perspectives. (7) The complexity of computation is greatly reduced compared with commonly used existing methods $[26,50]$ in the literature.
The rest of this paper is organized as follows: A comprehensive literature review on FDEA models is presented in "Literature review". In "Preliminaries", we review some necessary concepts and backgrounds on fuzzy arithmetic. In "The proposed method", we present a methodology for solving the FDEA models with undesirable outputs based on the positive ideal point (PIP) and negative ideal point (NIP) formulations, and then propose a fuzzy ranking algorithm for comparison and ranking of fuzzy efficiencies of DMUs. In "Numerical example", we solve a numerical example to demonstrate the performance of the proposed method. Finally, the conclusions are presented in "Concluding remarks".

\section{Literature review}

The fuzzy set theory introduced by Zadeh [78] is a general and natural extension of the ordinary set theory. Using the fuzzy concepts, the classic management techniques can be transformed into their fuzzy forms to be used more effectively in various management tasks such as decision making, policy making and planning. With the fuzzy management science, one can design a model that, like humans, would be capable of intelligent processing of qualitative information. Thus, the fuzzy management science allows us to introduce some flexibility to the classical crisp models by incorporating in them concept such as knowledge, experience and human judgment in order to achieve more practical solutions. In recent years, many researchers have worked on the implications and utility of imprecise and ambiguous qualitative data for DEA and performance evaluation in general. An overview of the papers which studied FDEA models and discussed regarding solution methodologies can be summarized as follows.

Sengupta [62] proposed a fuzzy programming approach to solve FDEA models where the objective function and constraints are not satisfied based on crisp definitions. Triantis et al. [68] fuzzified the non-radial DEA models for dealing with imprecise data. In this approach, the possible values of parameters of fuzzy programming model were considered as an interval, where the lower bound represents uncertainty and risk-free value and the upper bound represents the impossible value. Sheth [63] developed a method for the use of goal planning technique to solve DEA models in fuzzy environment. Cooper et al. [14] developed a model that utilizes a combination of imprecise data with known bounds and exact data of evaluation. Gu [24] modified the CCR model with symmetric TFNs. In this study, first the $\alpha$-cut method was applied on constraints and the obtained intervals were compared, and then the efficiency of units was evaluated by solving two linear programming problems. Saati et al. [59] proposed another approach to FDEA, where, instead of comparing equality or inequality of intervals, a variable defined within the interval is 
used to not only satisfy the constraints, but also optimize the efficiency values. Wu [74] and Wang and Luo [70] presented a method for ranking DMUs in FDEA based on the theory of ideal and anti-ideal units. Peykani et al. [49] proposed a novel fuzzy DEA model based on general fuzzy measure to consider the preferences of DMUs. Arya and Yadav [4] developed intuitionistic fuzzy DEA and dual intuitionistic fuzzy DEA to determine the intuitionistic fuzzy efficiencies of DMUs. Arana-Jiménez et al. [2] proposed a fuzzy DEA slacks-based approach to deals with the problem of efficiency assessment. Bagheri et al. [6] proposed a fuzzy data envelopment analysis approach for solving fuzzy multi objective transportation problem. Arana-Jiménez et al. [3] proposed a new radial, input-oriented and fully fuzzy DEA approach for assessing the relative efficiency of a set of DMUs. Singh and Pant [64] proposed a fuzzy DEA approach for analyzing the performance of eight selected paper mills of India. The currently available methods for solving FDEA models can be classified into six categories shown in Table 1.

An evaluation of the efficiency of DMUs should be able to account for the fact that some outputs and inputs may be desirable and others may be undesirable. A simple example of undesirable output is the number of defective products, which should be reduced. In the production processes, an inefficient unit that produces pollution and waste alongside the final product has two types of outputs (desirable and undesirable), of which one must be increased and the other must be reduced. This is slightly different from the general DEA models, and therefore requires a different approach to desirable and undesirable parameters. In such conditions, the model should be developed with the purpose of decreasing the undesirable parameters. Several models with undesirable inputs and outputs have already been developed to address such conditions. The notable works carried out in this area include the studies of Chung et al. [13], Ebrahimnejad [16], Färe et al. [21] Jahanshahloo et al. [29] Pathomsiri et al. [47], Seiford et al. [61], Nasseri et al. [43], Ebrahimnjead et al. [17, 18]. Scheel et al. [60] used the data transformation method to convert the undesirable elements to desirable elements while preserving the model linearity. According to Scheel et al. [60], the methods of aggregating undesirable outputs in DEA can be categorized into direct and indirect approaches. In the indirect approach, a monotonic decreasing function can be used to transform the values of undesirable outputs so that they could be treated as desirable outputs. In contrast, the direct approach means avoiding such data transformation and instead incorporating the undesired outputs directly into the DEA model. One of the direct methods of dealing with undesirable outputs is the approach proposed by Korhonen and Luptacik [35] that aggregates all desirable and undesirable outputs using a weighted sum operator. However, in this model, the weight factors of the undesirable outputs must be negative, so the relative efficiency of some DMUs could
Table 1 A brief summary of fuzzy DEA methods

\begin{tabular}{|c|c|}
\hline & The FDEA methods \\
\hline The tolerance approach & $\begin{array}{l}\text { Sengupta [62], Kahraman and } \\
\text { Tolga [30], Triantis and Girod } \\
\text { [68] }\end{array}$ \\
\hline The $\alpha$-level based approach & $\begin{array}{l}\text { Girod [23], Carlsson and } \\
\text { Korhonen [10], Noura and } \\
\text { Saljooghi [46], Noura et al. } \\
\text { [45], Kao and Lin [31], Kao } \\
\text { and Liu [33], Kao and Liu } \\
\text { [32], Kao and Liu [34], Saati } \\
\text { et al. [59], Saati and } \\
\text { Memariani [57], Saati and } \\
\text { Memariani [58], Saati et al. } \\
\text { [56], Chen and Klein's [12], } \\
\text { Hatami-Marbini and Saati } \\
\text { [25] }\end{array}$ \\
\hline The fuzzy ranking approach & $\begin{array}{l}\text { Guo and Tanaka [24], Ramík } \\
\text { and Rímánek [54], Tanaka } \\
\text { et al. [66], Beiranvand et al. } \\
\text { [9], Amindoust et al. [1], } \\
\text { Hosseinzadeh Lotfi et al. [27], } \\
\text { Soleimani-damaneh [65], } \\
\text { Zhou et al. [82], Noora and } \\
\text { Karami [44], Jahanshahloo } \\
\text { et al. [28], Emrouznejad et al. } \\
\text { [20] }\end{array}$ \\
\hline The possibility approach & $\begin{array}{l}\text { Zadeh [80], Dubois and Prade } \\
\text { [15], Payan and Sharifi [48], } \\
\text { Wen et al. [73], Lin [40], } \\
\text { Ramezanzadeh et al. [53], } \\
\text { Lertworasirikul et al. [37], } \\
\text { Lertworasirikul et al. [39], } \\
\text { Lertworasirikul et al. [38] }\end{array}$ \\
\hline The fuzzy arithmetic & $\begin{array}{l}\text { Wang et al. [69], Wang et al. } \\
\text { [71], Azadi et al. [5], } \\
\text { Mirhedayatian et al. [42], } \\
\text { Jafarian-Moghaddam and } \\
\text { Ghoseiri [26], Razavi } \\
\text { Hajiagha et al. [55] }\end{array}$ \\
\hline $\begin{array}{l}\text { The fuzzy random/type-2 fuzzy } \\
\text { set }\end{array}$ & $\begin{array}{l}\text { Zadeh [79], Kwakernaak [36], } \\
\text { Zerafat Angiz et al. [81], Qin } \\
\text { et al. [51], Qin et al. [52], } \\
\text { Tavana et al. [67] }\end{array}$ \\
\hline
\end{tabular}

become negative as well. Later, Puri and Yadav [50] modified this method to resolve this issue. We extend the DEA model with undesirable outputs proposed by Puri and Yadav [50] from the perspective of fuzzy arithmetic to deal with fuzziness in input and output data in DEA.

\section{Preliminaries}

This section is composed of three subsections to present some preliminaries about the fuzzy sets theory, DEA model with IDMU or ADMU and DEA model with undesirable outputs $[19,27]$. 


\section{Fuzzy set theory}

Definition 1 A fuzzy set $\tilde{A}$, defined on universal set of real numbers, $\mathbb{R}$, is said to be a fuzzy number if its membership function has the following characteristics:

- $\tilde{A}$ is convex, i.e., $\forall x, y \in \mathbb{R}, \forall \lambda \in[0,1]$, $\mu_{\tilde{A}}\left(\lambda x+(1-\lambda) y \geq \min \left\{\mu_{\tilde{A}}(x), \mu_{\tilde{A}}(\mathrm{y})\right\}\right.$,

- $\tilde{A}$ is normal, i.e., $\exists \bar{x} \in R ; \mu_{\tilde{A}}(\bar{x})=1$, and

- $\mu_{\tilde{A}}$ is piecewise continuous.

Definition 2 A triangular fuzzy number $\tilde{A}$, denoted by $\tilde{A}=$ $\left(a^{L}, a^{M}, a^{U}\right)$ is a fuzzy set on $\mathbb{R}$ with the membership function as follows:

$\mu_{\tilde{A}}(x)=\left\{\begin{array}{l}\frac{x-a^{L}}{a^{M}-a^{L}}, a^{L} \leq x \leq a^{M}, \\ \frac{a^{U}-x}{a^{U}-a^{M}}, a^{M} \leq x \leq a^{U} .\end{array}\right.$

We denote all triangular fuzzy numbers by $F(\mathbb{R})$.

Definition 3 A triangular fuzzy number $\tilde{A}=\left(a^{L}, a^{M}, a^{U}\right)$ is said to be a non-negative (respectively, positive) fuzzy number if, and only if, $a^{L} \geq 0$ (respectively, $a^{L}>0$ ).

Definition 4 Let $\tilde{A}=\left(a^{L}, a^{M}, a^{U}\right)$ and $\tilde{B}=\left(b^{L}, b^{M}\right.$, $b^{U}$ ) be two triangular fuzzy numbers and $k \in R$. Then, the arithmetic operations on $\tilde{A}$ and $\tilde{B}$ are defined as follows:

(i) $\tilde{A}+\tilde{B}=\left(a^{L}+b^{L}, a^{M}+b^{M}, a^{U}+b^{U}\right)$,

(ii) $\tilde{A}-\tilde{B}=\left(a^{L}-b^{U}, a^{M}-b^{M}, a^{U}-b^{L}\right)$,

(iii) $\tilde{A} \times \tilde{B} \approx\left(a^{L} b^{L}, a^{M} b^{M}, a^{U} b^{U}\right), a^{L}, b^{L} \geq 0$,

(iv) $\tilde{A} / \tilde{B} \approx\left(\frac{a^{L}}{b^{U}}, \frac{a^{M}}{b^{M}}, \frac{a^{U}}{b^{L}}\right), \quad a^{L} \geq 0, b^{L}>0$,

(v) $k \geq 0, k \tilde{A}=\left(k a^{L}, k a^{M}, k a^{U}\right)$,

(vi) $k<0, k \tilde{A}=\left(k a^{U}, k a^{M}, k a^{L}\right)$.

Ranking fuzzy numbers plays a very important role in decision-making, optimization, and other usages. The past few decades have seen a large number of approaches investigated for ranking fuzzy numbers $[72,75,76]$. Herein, we describe a novel ranking approach using integral values [41, 77].

Suppose there are $n$ fuzzy numbers $\tilde{A}_{i}=\left(a_{i}^{L}, a_{i}^{M}, a_{i}^{U}\right)$, $i=1,2, \ldots, n$, each with the left membership function $\mu_{\tilde{A}_{i}}^{L}(x)=\frac{x-a_{i}^{L}}{a_{i}^{U}-a_{i}^{L}}$ and right membership function $\mu_{\tilde{A}_{i}}^{R}(x)=$ $\frac{a_{i}^{U}-x}{a_{i}^{U}-a_{i}^{M}}$. The minimum crisp value $x_{\min }$ and the maximum crisp value $x_{\max }$ are defined as $x_{\min }=\inf \bigcup_{i=1}^{n}\left(a_{i}^{L}, a_{i}^{U}\right)$ and $x_{\max }=\sup \bigcup_{i=1}^{n}\left(a_{i}^{L}, a_{i}^{U}\right)$, respectively.
Definition 5 The left and right integral values of $\tilde{A}_{i}=\left(a_{i}^{L}\right.$, $a_{i}^{M}, a_{i}^{U}$ ) with regard to $x_{\min }$ are defined as follows $[41,77]$ :

$$
\begin{aligned}
S_{\min }^{L}\left(\tilde{A}_{i}\right) & =\left(a_{i}^{M}-x_{\min }\right)-\int_{a_{i}^{L}}^{a_{i}^{M}} \mu_{\tilde{A}_{i}}^{L}(x) \mathrm{d} x \\
& =\frac{a_{i}^{L}+a_{i}^{M}}{2}-x_{\min },
\end{aligned}
$$

$S_{\min }^{R}\left(\tilde{A}_{i}\right)=\left(a_{i}^{M}-x_{\min }\right)+\int_{a_{i}^{M}}^{a_{i}^{U}} \mu_{\tilde{A}_{i}}^{L}(x) \mathrm{d} x=\frac{a_{i}^{M}+a_{i}^{U}}{2}-x_{\min }$.

It should be noted that the fuzzy number $\tilde{A}_{i}$ becomes larger if $S_{\min }^{L}\left(\tilde{A}_{i}\right)$ and $S_{\min }^{R}\left(\tilde{A}_{i}\right)$ are larger.

Definition 6 The total integral value of $\tilde{A}_{i}$ with regard to $x_{\text {min }}$ with index of optimism $\alpha \in[0,1]$ is then defined as $[41,77]$ :

$$
\begin{aligned}
S_{\min }^{\alpha}\left(\tilde{A}_{i}\right) & =\alpha S_{\min }^{R}\left(\tilde{A}_{i}\right)+(1-\alpha) S_{\min }^{L}\left(\tilde{A}_{i}\right) \\
& =\frac{1}{2}\left[\alpha\left(a_{i}^{M}+a_{i}^{U}\right)+(1-\alpha)\left(a_{i}^{L}+a_{i}^{M}\right)-2 x_{\min }\right] .
\end{aligned}
$$

Definition 7 The left and right integral values of $\tilde{A}_{i}=\left(a_{i}^{L}\right.$, $a_{i}^{M}, a_{i}^{U}$ ) with regard to $x_{\max }$ are defined as follows:

$$
\begin{aligned}
S_{\max }^{L}\left(\tilde{A}_{i}\right) & =\left(x_{\max }-a_{i}^{M}\right)+\int_{a_{i}^{L}}^{a_{i}^{M}} \mu_{\tilde{A}_{i}}^{L}(x) \mathrm{d} x \\
& =x_{\max }-\frac{a_{i}^{L}+a_{i}^{M}}{2}, \\
S_{\max }^{R}\left(\tilde{A}_{i}\right) & =\left(x_{\max }-a_{i}^{M}\right)-\int_{a_{i}^{M}}^{a_{i}^{U}} \mu_{\tilde{A}_{i}}^{R}(x) \mathrm{d} x \\
& =x_{\max }-\frac{a_{i}^{M}+a_{i}^{U}}{2} .
\end{aligned}
$$

It should be noted that the fuzzy number $\tilde{A}_{i}$ becomes larger if $S_{\max }^{L}\left(\tilde{A}_{i}\right)$ and $S_{\max }^{R}\left(\tilde{A}_{i}\right)$ are smaller.

Definition 8 The total integral value of $\tilde{A}_{i}$ with regard to $x_{\max }$ with index of optimism $\alpha \in[0,1]$ is then defined as follows:

$$
\begin{aligned}
& S_{\max }^{\alpha}\left(\tilde{A}_{i}\right) \\
& \quad=\alpha S_{\max }^{R}\left(\tilde{A}_{i}\right)+(1-\alpha) S_{\max }^{L}\left(\tilde{A}_{i}\right) \\
& \quad=\frac{1}{2}\left[2 x_{\max }-\left(\alpha\left(a_{i}^{M}+a_{i}^{U}\right)+(1-\alpha)\left(a_{i}^{L}+a_{i}^{M}\right)\right)\right] .
\end{aligned}
$$


The two distinctive integral values (3) and (5) are combined to form a comprehensive index called the relative closeness (RC) to the total integral value of $\tilde{A}_{i}$ with regard to $x_{\min }$ just like the well-known TOPSIS approach in multiple attribute decision making (MADM) as follows:

$$
\begin{aligned}
& \operatorname{RC}^{\alpha}\left(\tilde{A}_{i}\right) \\
& =\frac{S_{\text {min }}^{\alpha}\left(\tilde{A}_{i}\right)}{S_{\max }^{\alpha}\left(\tilde{A}_{i}\right)+S_{\min }^{\alpha}\left(\tilde{A}_{i}\right)} \\
& \quad=\frac{1}{2}\left[\frac{\alpha\left(a_{i}^{M}+a_{i}^{U}\right)+(1-\alpha)\left(a_{i}^{L}+a_{i}^{M}\right)-2 x_{\min }}{x_{\max }-x_{\min }}\right] .
\end{aligned}
$$

The proposed approach uses $\operatorname{RC}^{\alpha}\left(\tilde{A}_{i}\right)$ to rank fuzzy numbers. The reason is that the more distance between the fuzzy number $\tilde{A}_{i}$ and $x_{\min }$ measured by $S_{\min }^{\alpha}\left(\tilde{A}_{i}\right)$ indicates the higher rank of $\tilde{A}_{i}$ and the less distance between the fuzzy number $\tilde{A}_{i}$ and $x_{\max }$ measured by $S_{\max }^{\alpha}\left(\tilde{A}_{i}\right)$ indicates the higher rank of $\tilde{A}_{i}$. Therefore, the larger $\operatorname{RC}^{\alpha}\left(\tilde{A}_{i}\right)$ is, the larger is the fuzzy number $\tilde{A}_{i}$.

It is worth noting that the basic idea of TOPSIS originates from the concept of a displaced ideal point from which the compromise solution has the shortest distance. It will be based on the shortest distance from the ideal and the farthest from the anti-ideal solution. TOPSIS simultaneously considers the distances to both ideal and ant-ideal solutions, and a preference order is ranked according to their relative closeness, and a combination of these two distance measures. With this in mind, the main advantages of the suggested relative closeness index defined in Eq. (7) over other alternatives are (1) giving a scalar value that accounts for both the best and worst alternatives simultaneously, (2) being a simple computation process that can be easily programmed into a spreadsheet, (3) visualizing the performance measures of all alternatives on attributes at least for any two dimensions, and (4) posing a sound logic that represents the rationale of human choice.

\section{DEA model with IDMU or ADMU}

In this subsection, we review DEA models with IDMU and ADMU that evaluate DMUs from the viewpoint of the best and worst possible relative efficiency, respectively [70, 74].

Consider a set of $n$ DMUs, where DMUj consumes $m$ inputs $x_{i j}(i=1, \ldots, m)$ to produce $r$ outputs $y_{r j}(r=1$, $\ldots, s)$. An IDMU is a hypothetical DMU which consumes the least inputs to generate the most outputs. An ADMU is a hypothetical DMU which consumes the most inputs to generate the least outputs. The inputs and outputs of IDMU =
$\left(X^{\min }, Y^{\max }\right)$ and ADMU $=\left(X^{\max }, Y^{\min }\right)$ are defined as follows:

$$
\begin{aligned}
& x_{i}^{\min }=\min _{j}\left\{x_{i j}\right\}, \quad x_{i}^{\max }=\max _{j}\left\{x_{i j}\right\}, \quad i=1, \ldots, m \\
& y_{r}^{\max }=\max _{j}\left\{y_{r j}\right\}, \quad y_{r}^{\min }=\min _{j}\left\{y_{r j}\right\}, \quad r=1, \ldots, s .
\end{aligned}
$$

The best relative efficiency of IDMU $\left(\theta_{I}^{*}\right)$ is computed by solving the following fractional programming model:

$$
\begin{aligned}
& \theta_{I}^{*}=\max \frac{\sum_{r=1}^{s} u_{r} y_{r}^{\max }}{\sum_{i=1}^{m} v_{i} x_{i}^{\min }} \\
& \text { s.t. } \theta_{j}=\frac{\sum_{r=1}^{s} u_{r} y_{r j}}{\sum_{i=1}^{m} v_{i} x_{i j}} \leq 1 \quad j=1,2, \ldots, n \\
& u_{r} \geq \varepsilon \forall r, \quad \mathrm{v}_{i} \geq \varepsilon \forall i .
\end{aligned}
$$

The worst relative efficiency of $\operatorname{ADMU}\left(\varphi_{N}^{*}\right)$ is computed by solving the following fractional programming model:

$$
\begin{aligned}
\varphi_{N}^{*} & =\min \frac{\sum_{r=1}^{s} u_{r} y_{r}^{\min }}{\sum_{i=1}^{m} v_{i} x_{i}^{\max }} \\
\text { s.t. } & \frac{\sum_{r=1}^{s} u_{r} y_{r}^{\max }}{\sum_{i=1}^{m} v_{i} x_{i}^{\min }} \geq \theta_{I}^{*} \\
\varphi_{j} & =\frac{\sum_{r=1}^{s} u_{r} y_{r j}}{\sum_{i=1}^{m} v_{i} x_{i j}} \leq 1 \quad j=1,2, \ldots, n
\end{aligned}
$$$$
u_{r} \geq \varepsilon \forall r, \quad v_{i} \geq \varepsilon \forall i \text {. }
$$

The best relative efficiency of $\mathrm{DMU}_{o}\left(\theta_{o}^{*}\right)$ using the optimum efficiency of IDMU is computed by solving the following fractional programming model:

$\theta_{o}^{*}=\operatorname{Max} \frac{\sum_{r=1}^{s} u_{r} y_{r o}}{\sum_{i=1}^{m} v_{i} x_{i o}}$

s.t. $\frac{\sum_{r=1}^{s} u_{r} y_{r}^{\max }}{\sum_{i=1}^{m} v_{i} x_{i}^{\min }}=\theta_{I}^{*}$

$\theta_{j}=\frac{\sum_{r=1}^{s} u_{r} y_{r j}}{\sum_{i=1}^{m} v_{i} x_{i j}} \leq 1 \quad j=1,2, \ldots . n$

$u_{r} \geq \varepsilon \forall r, \quad v_{i} \geq \varepsilon \forall i$.

The worst relative efficiency of $\operatorname{DMU}_{o}\left(\varphi_{o}^{*}\right)$ using the optimum efficiency of ADMU is computed by solving the following fractional programming model:

$$
\begin{aligned}
\varphi_{o}^{*} & =\min \frac{\sum_{r=1}^{s} u_{r} y_{r o}}{\sum_{i=1}^{m} v_{i} x_{i o}} \\
\text { s.t. } & \frac{\sum_{r=1}^{s} u_{r} y_{r}^{\min }}{\sum_{i=1}^{m} v_{i} x_{i}^{\max }}=\varphi_{N}^{*} \\
\varphi_{j} & =\frac{\sum_{r=1}^{s} u_{r} y_{r j}}{\sum_{i=1}^{m} v_{i} x_{i j}} \leq 1 \quad j=1,2, \ldots, n
\end{aligned}
$$


$u_{r} \geq \varepsilon \forall r, \quad v_{i} \geq \varepsilon \forall i$.

Remark 1 The aim of model (11) is to maximize the efficiency of each DMU while at the same time keep the maximal efficiency of the IDMU unchanged. In as same way, the aim of model (12) to minimize the efficiency of each DMU under the condition that the optimal efficiency of the ADMU keeps unchanged. Wang and Luo [70] and Wu [74] integrated the optimal values of models (8)-(12) to provide an overall assessment of each DMU as follows:

$\mathrm{RC}_{o}=\frac{\varphi_{o}^{*}-\varphi_{N}^{*}}{\left(\varphi_{o}^{*}-\varphi_{N}^{*}\right)+\left(\theta_{I}^{*}-\theta_{o}^{*}\right)}$

It is obvious that the bigger $\varphi_{o}^{*}-\varphi_{N}^{*}$ (difference of efficiencies of DMU under consideration and ADMU) and the smaller $\theta_{I}^{*}-\theta_{o}^{*}$ (difference of efficiencies of IDMU and DMU under consideration) mean the better performance of DMU under evaluation. Hence, the bigger is the $\mathrm{RC}_{o}$ value, the better is the performance of under evaluation $\mathrm{DMU}_{o}$.

\section{DEA model with undesirable outputs}

In this subsection, we review the DEA model developed by Puri and Yadav [50] to deal with undesirable outputs.

Consider a set of $n$ DMUs, where $\mathrm{DMU}_{j}$ consumes $m$ inputs $x_{i j}(i=1, \ldots, m)$ to produce $s$ outputs in which $s_{1}$ outputs are desirable (good) and $s_{2}$ outputs are undesirable (bad) such that $s=s_{1}+s_{2}$. The desirable and undesirable outputs are denoted by $y_{r j}^{g}\left(r=1, \ldots, s_{1}\right)$ and $y_{p j}^{b}\left(p=1, \ldots, s_{2}\right)$, respectively. Puri and Yadav [50] modified the model suggested by Korhonen and Luptacik [35] to that with undesirable outputs and proposed the following fractional programming model to determine the efficiency of each DMU in the presence of undesirable outputs:

$\max E_{o}=\frac{\sum_{r=1}^{s_{1}} u_{r}^{g} y_{r o}^{g}-\sum_{p=1}^{s_{2}} u_{p}^{b} y_{p o}^{b}}{\sum_{i=1}^{m} v_{i} x_{i o}}$

s.t. $0 \leq E_{j}=\frac{\sum_{r=1}^{s_{1}} u_{r}^{g} y_{r j}^{g}-\sum_{p=1}^{s_{2}} u_{p}^{b} y_{p j}^{b}}{\sum_{i=1}^{m} v_{i} x_{i j}} \leq 1 \quad j=1, \ldots, n$

$u_{r}^{g} \geq \varepsilon \forall r, \quad u_{p}^{b} \geq \varepsilon \forall p, \quad v_{i} \geq \varepsilon \forall i$.

The model (13) is transformed into the following linear programming model by using Charnes-Cooper transformation: $\min E_{o}=\sum_{r=1}^{s_{1}} u_{r}^{g} y_{r o}^{g}-\sum_{p=1}^{s_{2}} u_{p}^{b} y_{p o}^{b}$

s.t. $\sum_{i=1}^{m} v_{i} x_{i o}=1$

$\sum_{r=1}^{s_{1}} u_{r}^{g} y_{r j}^{g}-\sum_{p=1}^{s_{2}} u_{p}^{b} y_{p j}^{b}-\sum_{i=1}^{m} v_{i} x_{i j} \leq 0 \quad j=1, \ldots, n$,

$\sum_{r=1}^{s_{1}} u_{r}^{g} y_{r j}^{g}-\sum_{p=1}^{s_{2}} u_{p}^{b} y_{p j}^{b} \geq 0 \quad j=1, \ldots, n$,

$u_{r}^{g} \geq \varepsilon \forall r, \quad u_{p}^{b} \geq \varepsilon \forall p, \quad v_{i} \geq \varepsilon \forall i$.

\section{The proposed method}

In this section, we first develop a new DEA model with IDMU and ADMU in the presence of undesirable outputs and then the proposed model is extended into a fuzzy environment.

\section{DEA model with undesirable outputs based on IDMU and ADMU}

In this subsection, we propose a new methodology for assessing the efficiency of each DMU which is inspired by the work of Puri and Yadav [50] on considering undesirable outputs and the work of $\mathrm{Wu}$ [74] on considering virtual DMUs.

The inputs and desirable and undesirable outputs of $\mathrm{IDMU}=\left(X^{\min }, Y^{g \max }, Y^{b \max }\right)$ and $\mathrm{ADMU}=\left(X^{\max }\right.$, $\left.Y^{\text {gmin }}, Y^{\text {minin }}\right)$ are defined as follows:

$$
\begin{aligned}
x_{i}^{\min }=\min _{j}\left\{x_{i j}\right\}, \quad x_{i}^{\max }=\max _{j}\left\{x_{i j}\right\}, & i=1, \ldots, m, \\
y_{r}^{g \max }=\max _{j}\left\{y_{r j}^{g}\right\}, \quad y_{r}^{g \min }=\min _{j}\left\{y_{r j}^{g}\right\}, & r=1, \ldots, s_{1}, \\
y_{p}^{b \max }=\max _{j}\left\{y_{p j}^{b}\right\}, \quad y_{p}^{b \min }=\min _{j}\left\{y_{p j}^{b}\right\}, & p=1, \ldots, s_{2} .
\end{aligned}
$$

The best relative efficiency of IDMU $\left(\theta_{I}^{*}\right)$ in the presence of undesirable outputs is computed by solving the following model: 
$\theta_{I}^{*}=\max \frac{\sum_{r=1}^{s_{1}} u_{r}^{g} y_{r}^{g \max }-\sum_{p=1}^{s_{2}} u_{p}^{b} y_{p}^{b \max }}{\sum_{i=1}^{m} v_{i} x_{i}^{\min }}$

s.t. $\theta_{j}=\frac{\sum_{r=1}^{s_{1}} u_{r}^{g} y_{r j}^{g}-\sum_{p=1}^{s_{2}} u_{p}^{b} y_{p j}^{b}}{\sum_{i=1}^{m} v_{i} x_{i j}} \leq 1, \quad j=1, \ldots, n$,

$\sum_{r=1}^{s_{1}} u_{r}^{g} y_{r j}^{g}-\sum_{p=1}^{s_{2}} u_{p}^{b} y_{p j}^{b} \geq 0, \quad j=1, \ldots, n$

$\sum_{r=1}^{s_{1}} u_{r}^{g} y_{r}^{g \max }-\sum_{p=1}^{s_{2}} u_{p}^{b} y_{p}^{b \max } \geq 0$,

$u_{r}^{g} \geq \varepsilon \forall r, \quad u_{p}^{b} \geq \varepsilon \forall p, \quad v_{i} \geq \varepsilon \forall i$.

It should be noted that the second constraint set ensures the non-negativity efficiency of each DMU and the third constrain ensures the non-negativity efficiency of IDMU.

The worst relative efficiency of $\operatorname{ADMU}\left(\varphi_{N}^{*}\right)$ in the presence of undesirable outputs is computed by solving the following model:

$$
\begin{aligned}
& \varphi_{N}^{*}=\min \frac{\sum_{r=1}^{s_{1}} u_{r}^{g} y_{r}^{g \min }-\sum_{p=1}^{s_{2}} u_{p}^{b} y_{p}^{b \min }}{\sum_{i=1}^{m} v_{i} x_{i}^{\max }} \\
& \text { s.t. } \frac{\sum_{r=1}^{s_{1}} u_{r}^{g} y_{r}^{g \max }-\sum_{p=1}^{s_{2}} u_{p}^{b} y_{p}^{b \max }}{\sum_{i=1}^{m} v_{i} x_{i}^{\min }} \geq \theta_{I}^{*} \\
& \varphi_{j}=\frac{\sum_{r=1}^{s_{1}} u_{r}^{g} y_{r j}^{g}-\sum_{p=1}^{s_{2}} u_{p}^{b} y_{p j}^{b}}{\sum_{i=1}^{m} v_{i} x_{i j}} \leq 1, \quad j=1, \ldots, n, \\
& \sum_{r=1}^{s_{1}} u_{r}^{g} y_{r j}^{g}-\sum_{p=1}^{s_{2}} u_{p}^{b} y_{p j}^{b} \geq 0, \quad j=1, \ldots, n, \\
& \sum_{r=1}^{s_{1}} u_{r}^{g} y_{r}^{g \min }-\sum_{p=1}^{s_{2}} u_{p}^{b} y_{p}^{b \min } \geq 0, \\
& u_{r}^{g} \geq \varepsilon \forall r, \quad u_{p}^{b} \geq \varepsilon \forall p, \quad v_{i} \geq \varepsilon \forall i .
\end{aligned}
$$

Similarly, the third constraint set ensures the nonnegativity efficiency of each DMU and the fourth constrain ensures the non-negativity efficiency of ADMU.

The best relative efficiency of $\operatorname{DMU}_{o}\left(\theta_{o}^{*}\right)$ in the presence of undesirable outputs using the optimum efficiency of IDMU is computed by solving the following model:

$$
\begin{aligned}
& \theta_{o}^{*}=\max \frac{\sum_{r=1}^{s_{1}} u_{r}^{g} y_{r o}^{g}-\sum_{p=1}^{s_{2}} u_{p}^{b} y_{p o}^{b}}{\sum_{i=1}^{m} v_{i} x_{i o}} \\
& \text { s.t. } \frac{\sum_{r=1}^{s_{1}} u_{r}^{g} y_{r}^{g \max }-\sum_{p=1}^{s_{2}} u_{p}^{b} y_{p}^{b \max }}{\sum_{i=1}^{m} v_{i} x_{i}^{\min }}=\theta_{I}^{*} \\
& \theta_{j}=\frac{\sum_{r=1}^{s_{1}} u_{r}^{g} y_{r j}^{g}-\sum_{p=1}^{s_{2}} u_{p}^{b} y_{p j}^{b}}{\sum_{i=1}^{m} v_{i} x_{i j}} \leq 1, \quad j=1, \ldots, n, \\
& \sum_{r=1}^{s_{1}} u_{r}^{g} y_{r j}^{g}-\sum_{p=1}^{s_{2}} u_{p}^{b} y_{p j}^{b} \geq 0, \quad j=1, \ldots, n,
\end{aligned}
$$

$u_{r}^{g} \geq \varepsilon \forall r, \quad u_{p}^{b} \geq \varepsilon \forall p, \quad v_{i} \geq \varepsilon \forall i$.

The worst relative efficiency of $\mathrm{DMU}_{o}\left(\varphi_{o}^{*}\right)$ in the presence of undesirable outputs using the optimum efficiency of ADMU is computed by solving the following model:

$\varphi_{o}^{*}=\min \frac{\sum_{r=1}^{s_{1}} u_{r}^{g} y_{r o}^{g}-\sum_{p=1}^{s_{2}} u_{p}^{b} y_{p o}^{b}}{\sum_{i=1}^{m} v_{i} x_{i o}}$

s.t. $\frac{\sum_{r=1}^{s_{1}} u_{r}^{g} y_{r}^{g \min }-\sum_{p=1}^{s_{2}} u_{p}^{b} y_{p}^{b \min }}{\sum_{i=1}^{m} v_{i} x_{i}^{\max }}=\varphi_{N}^{*}$

$\varphi_{j}=\frac{\sum_{r=1}^{s_{1}} u_{r}^{g} y_{r j}^{g}-\sum_{p=1}^{s_{2}} u_{p}^{b} y_{p j}^{b}}{\sum_{i=1}^{m} v_{i} x_{i j}} \leq 1, \quad j=1, \ldots, n$

$\sum_{r=1}^{s_{1}} u_{r}^{g} y_{r j}^{g}-\sum_{p=1}^{s_{2}} u_{p}^{b} y_{p j}^{b} \geq 0, \quad j=1, \ldots, n$

$u_{r}^{g} \geq \varepsilon \forall r, \quad u_{p}^{b} \geq \varepsilon \forall p, \quad v_{i} \geq \varepsilon \forall i$.

By using Charnes-Cooper transformation, the model (16) is transformed into the following linear programming problem:

$\theta_{I}^{*}=\max \sum_{r=1}^{s_{1}} u_{r}^{g} y_{r}^{g \max }-\sum_{p=1}^{s_{2}} u_{p}^{b} y_{p}^{b \max }$

s.t. $\sum_{i=1}^{m} v_{i} x_{i}^{\min }=1$

$\sum_{r=1}^{s_{1}} u_{r}^{g} y_{r j}^{g}-\sum_{p=1}^{s_{2}} u_{p}^{b} y_{p j}^{b}-\sum_{i=1}^{m} v_{i} x_{i j} \leq 0, j=1, \ldots, n$,

$\sum_{r=1}^{s_{1}} u_{r}^{g} y_{r j}^{g}-\sum_{p=1}^{s_{2}} u_{p}^{b} y_{p j}^{b} \geq 0, \quad j=1, \ldots, n$,

$\sum_{r=1}^{s_{1}} u_{r}^{g} y_{r}^{g \max }-\sum_{p=1}^{s_{2}} u_{p}^{b} y_{p}^{b \max } \geq 0$,

$u_{r}^{g} \geq \varepsilon \forall r, \quad u_{p}^{b} \geq \varepsilon \forall p, \quad v_{i} \geq \varepsilon \forall i$.

Similarly, the model (17) is transformed into the following linear programming problem:

$$
\begin{aligned}
& \varphi_{N}^{*}=\min \sum_{r=1}^{s_{1}} u_{r}^{g} y_{r}^{g \min }-\sum_{p=1}^{s_{2}} u_{p}^{b} y_{p}^{b \min } \\
& \text { s.t. } \sum_{i=1}^{m} v_{i} x_{i}^{\max }=1 \\
& \sum_{r=1}^{s_{1}} u_{r}^{g} y_{r}^{g \max }-\sum_{p=1}^{s_{2}} u_{p}^{b} y_{p}^{b \max }-\theta_{I}^{*} \sum_{i=1}^{m} v_{i} x_{i}^{\min } \geq 0 \\
& \sum_{r=1}^{s_{1}} u_{r}^{g} y_{r j}^{g}-\sum_{p=1}^{s_{2}} u_{p}^{b} y_{p j}^{b}-\sum_{i=1}^{m} v_{i} x_{i j} \leq 0, \quad j=1, \ldots, n,
\end{aligned}
$$


$\sum_{r=1}^{s_{1}} u_{r}^{g} y_{r j}^{g}-\sum_{p=1}^{s_{2}} u_{p}^{b} y_{p j}^{b} \geq 0, \quad j=1, \ldots, n$,

$\sum_{r=1}^{s_{1}} u_{r}^{g} y_{r}^{g \min }-\sum_{p=1}^{s_{2}} u_{p}^{b} y_{p}^{b \min } \geq 0$,

$u_{r}^{g} \geq \varepsilon \forall r, \quad u_{p}^{b} \geq \varepsilon \forall p, \quad v_{i} \geq \varepsilon \forall i$.

In a similar way, the fractional programming models (18) and (19) are transformed into equivalent linear programming models.

Remark 2 It is required to point out that according to the proposed approach to deal with undesirable outputs, negative weights are taken for undesirable outputs. Thus, models (16) and (18) confirm that the IDMU consumes the minimal amount of inputs and produces the maximum amount of desirable outputs and the minimal amount of undesirable outputs. In a similar way, models (17) and (19) confirm that the ADMU consumes the maximum amount of inputs and produces the minimum amount of desirable inputs and the maximum amount of undesirable outputs.

\section{New fuzzy DEA models}

In this subsection, the proposed DEA model with IDMU and ADMU in the presence of undesirable outputs is extended to a fuzzy environment.

Let us assume all fuzzy inputs and outputs are triangular fuzzy numbers. Thus, fuzzy inputs $\tilde{x}_{i j}(i=1, \ldots, m)$, fuzzy desirable outputs $\tilde{y}_{r j}^{g}\left(r=1, \ldots, s_{1}\right)$ and fuzzy undesirable outputs $\tilde{y}_{p j}^{b}\left(p=1, \ldots, s_{2}\right)$ are represented by $\tilde{x}_{i j}=\left(x_{i j}^{L}\right.$, $\left.x_{i j}^{M}, x_{i j}^{U}\right), \tilde{y}_{r j}^{g}=\left(y_{r j}^{L g}, y_{r j}^{M g}, y_{r j}^{U g}\right)$ and $\tilde{y}_{p j}^{b}=\left(y_{p j}^{L b}, y_{p j}^{M b}\right.$, $\left.y_{p j}^{U b}\right)$, respectively. The fuzzy efficiency of $\mathrm{DMU}_{j}$ is defined as follows:

$\tilde{\theta}_{j}=\frac{\sum_{r=1}^{s_{1}} u_{r}^{g} \tilde{y}_{r j}^{g}-\sum_{p=1}^{s_{2}} u_{p}^{b} \tilde{y}_{p j}^{b}}{\sum_{i=1}^{m} v_{i} \tilde{x}_{i j}}$

The fuzzy inputs and fuzzy desirable and undesirable outputs of fuzzy IDMU, i.e., FIDMU $=\left(\tilde{X}^{\min }, \tilde{Y}^{g \max }, \tilde{Y}^{b \max }\right)$ and fuzzy ADMU, i.e., FADMU $=\left(\tilde{X}^{\max }, \tilde{Y}^{g \min }, \tilde{Y}^{b \min }\right)$ are defined as follows:

$$
\begin{aligned}
& \tilde{x}_{i}^{\min }=\left(x_{i}^{L \min }, x_{i}^{M \min }, x_{i}^{U \min }\right) \\
& =\left(\min _{j}\left\{x_{i j}^{L}\right\}, \min _{j}\left\{x_{i j}^{M}\right\}, \min _{j}\left\{x_{i j}^{U}\right\}\right), \\
& i=1, \ldots, m \text {, } \\
& x_{i}^{\max }=\left(x_{i}^{\operatorname{Lmax}}, x_{i}^{\operatorname{Mmax}}, x_{i}^{U \max }\right) \\
& =\left(\max _{j}\left\{x_{i j}^{L}\right\}, \max _{j}\left\{x_{i j}^{M}\right\}, \max _{j}\left\{x_{i j}^{U}\right\}\right) \text {, } \\
& i=1, \ldots, m \text {, } \\
& \tilde{y}_{r}^{g \max }=\left(y_{r}^{L g \max }, y_{r}^{M g \max }, y_{r}^{U g \max }\right) \\
& =\left(\max _{j}\left\{y_{r j}^{L g}\right\}, \max _{j}\left\{y_{r j}^{M g}\right\}, \max _{j}\left\{y_{r j}^{U g}\right\}\right) \text {, } \\
& r=1, \ldots, s_{1} \text {, } \\
& \tilde{y}_{r}^{g \min }=\left(y_{r}^{L g \min }, y_{r}^{M g \min }, y_{r}^{U g \min }\right) \\
& =\left(\min _{j}\left\{y_{r j}^{L g}\right\}, \min _{j}\left\{y_{r j}^{M g}\right\}, \min _{j}\left\{y_{r j}^{U g}\right\}\right), \\
& r=1, \ldots, s_{1} \text {, } \\
& \tilde{y}_{p}^{b \max }=\left(y_{p}^{L b \max }, y_{p}^{M b \max }, y_{p}^{U b \max }\right) \\
& =\left(\max _{j}\left\{y_{p j}^{L b}\right\}, \max _{j}\left\{y_{p j}^{M b}\right\}, \max _{j}\left\{y_{p j}^{U b}\right\}\right) \text {, } \\
& p=1, \ldots, s_{2} \text {, } \\
& \tilde{y}_{p}^{b \min }=\left(y_{p}^{L b \min }, y_{p}^{M b \min }, y_{p}^{U b \min }\right) \\
& =\left(\min _{j}\left\{y_{p j}^{L b}\right\}, \min _{j}\left\{y_{r p}^{M b}\right\}, \min _{j}\left\{y_{p j}^{U b}\right\}\right) \text {, } \\
& p=1, \ldots, s_{2} \text {. }
\end{aligned}
$$

The fuzzy efficiency of FIDMU and FADMU are defined as follows:

$\tilde{\theta}_{I}=\frac{\sum_{r=1}^{s_{1}} u_{r}^{g} \tilde{y}_{r}^{g \max }-\sum_{p=1}^{s_{2}} u_{p}^{b} \tilde{y}_{p}^{b \max }}{\sum_{i=1}^{m} v_{i} \tilde{x}_{i}^{\min }}$,
$\tilde{\varphi}_{N}=\frac{\sum_{r=1}^{s_{1}} u_{r}^{g} \tilde{y}_{r}^{g \min }-\sum_{p=1}^{s_{2}} u_{p}^{b} \tilde{y}_{p}^{b \min }}{\sum_{i=1}^{m} v_{i} \tilde{x}_{i}^{\max }}$.

\section{The best fuzzy efficiency of FIDMU}

Here, we describe a method to fine the best fuzzy efficiency of FIDMU.

The following model can be used to determine the best fuzzy relative efficiency of $\operatorname{FIDMU}\left(\tilde{\theta}_{I}^{*}\right)$ : 
$\tilde{\theta}_{I}^{*}=\max \frac{\sum_{r=1}^{s_{1}} u_{r}^{g} \tilde{y}_{r}^{g \max }-\sum_{p=1}^{s_{2}} u_{p}^{b} \tilde{y}_{p}^{b \max }}{\sum_{i=1}^{m} v_{i} \tilde{x}_{i}^{\min }}$

s.t. $\tilde{\theta}_{j}=\frac{\sum_{r=1}^{s_{1}} u_{r}^{g} \tilde{y}_{r j}^{g}-\sum_{p=1}^{s_{2}} u_{p}^{b} \tilde{y}_{p j}^{b}}{\sum_{i=1}^{m} v_{i} \tilde{x}_{i j}} \leq 1, j=1, \ldots, n$,

$\sum_{r=1}^{s_{1}} u_{r}^{g} \tilde{y}_{r j}^{g}-\sum_{p=1}^{s_{2}} u_{p}^{b} \tilde{y}_{p j}^{b} \geq 0, j=1, \ldots, n$

$\sum_{r=1}^{s_{1}} u_{r}^{g} \tilde{y}_{r}^{g \max }-\sum_{p=1}^{s_{2}} u_{p}^{b} \tilde{y}_{p}^{b \max } \geq 0$

$u_{r}^{g} \geq \varepsilon \forall r, \quad u_{p}^{b} \geq \varepsilon \forall p, \quad v_{i} \geq \varepsilon \forall i$.

Here, we use a modification of the method proposed by Wang et al. [71] for solving the fuzzy model (26) which is based on fuzzy arithmetic operations. By using triangular fuzzy numbers, the fuzzy model (26) is formulated as follows:
In the model (28), as long as upper bound $\theta_{I}^{U}$ is kept to be less than or equal to one, $\theta_{I}^{M}$ and $\theta_{I}^{L}$ will be spontaneously satisfied. Similarly, as long as lower bound $\sum_{r=1}^{s_{1}} u_{r}^{g} y_{r j}^{L g}-\sum_{p=1}^{s_{2}} u_{p}^{b} y_{p j}^{U b}$ is kept to be more than or equal to zero, then $\sum_{r=1}^{s_{1}} u_{r}^{g} y_{r j}^{M g}-\sum_{p=1}^{s_{2}} u_{p}^{b} y_{p j}^{M b}$ and $\sum_{r=1}^{s_{1}} u_{r}^{g} y_{r j}^{U g}-\sum_{p=1}^{s_{2}} u_{p}^{b} y_{p j}^{L b}$ will be spontaneously satisfied. Moreover, as long as lower bound $\sum_{r=1}^{s_{1}} u_{r}^{g} y_{r}^{L g m a x}-$ $\sum_{p=1}^{s_{2}} u_{p}^{b} y_{p}^{U b \max }$ is kept to be more than or equal to zero, $\sum_{r=1}^{s_{1}} u_{r}^{g} y_{r}^{M g \max }-\sum_{p=1}^{s_{2}} u_{p}^{b} y_{p}^{M b \max }$ and $\sum_{r=1}^{s_{1}} u_{r}^{g} y_{r}^{U g \max }-$ $\sum_{p=1}^{s_{2}} u_{p}^{b} y_{p}^{L b \max }$ will be spontaneously satisfied. Therefore, the model (28) is simplified as follows:

$$
\begin{aligned}
& \tilde{\theta}_{I}^{*}=\max \tilde{\theta}_{I} \approx\left[\theta_{I}^{L}, \theta_{I}^{M}, \theta_{I}^{U}\right]=\frac{\sum_{r=1}^{s_{1}} u_{r}^{g}\left(y_{r}^{L g \max }, y_{r}^{M g \max }, y_{r}^{U g \max }\right)-\sum_{p=1}^{s_{2}} u_{p}^{b}\left(y_{p}^{L b \max }, y_{p}^{M b \max }, y_{p}^{U b \max }\right)}{\sum_{i=1}^{m} v_{i}\left(x_{i}^{L \min }, x_{i}^{M \min }, x_{i}^{U \min }\right)} \\
& \text { s.t. } \tilde{\theta}_{j}=\frac{\sum_{r=1}^{s_{1}} u_{r}^{g}\left(y_{r j}^{g L}, y_{r j}^{g M}, y_{r j}^{g U}\right)-\sum_{p=1}^{s_{2}} u_{p}^{b}\left(y_{p j}^{b L}, y_{p j}^{b M}, y_{p j}^{b U}\right)}{\sum_{i=1}^{m} v_{i}\left(x_{i j}^{L}, x_{i j}^{M}, x_{i j}^{U}\right)} \leq 1, j=1, \ldots, n, \\
& \sum_{r=1}^{s_{1}} u_{r}^{g}\left(y_{r j}^{g L}, y_{r j}^{g M}, y_{r j}^{g U}\right)-\sum_{p=1}^{s_{2}} u_{p}^{b}\left(y_{p j}^{b L}, y_{p j}^{b M}, y_{p j}^{b U}\right) \geq 0, j=1, \ldots, n \\
& \sum_{r=1}^{s_{1}} u_{r}^{g}\left(y_{r}^{L g \max }, y_{r}^{M g \max }, y_{r}^{U g \max }\right)-\sum_{p=1}^{s_{2}} u_{p}^{b}\left(y_{p}^{L b \max }, y_{p}^{M b \max }, y_{p}^{U b \max }\right) \geq 0, \\
& u_{r}^{g} \geq \varepsilon \forall r, \quad u_{p}^{b} \geq \varepsilon \forall p, \quad v_{i} \geq \varepsilon \forall i .
\end{aligned}
$$

According to fuzzy arithmetic, the fuzzy model (27) is rewritten as follows:

$$
\begin{aligned}
& \tilde{\theta}_{I}^{*}=\max \tilde{\theta}_{I} \approx\left[\theta_{I}^{L}, \theta_{I}^{M}, \theta_{I}^{U}\right] \\
& =\left[\frac{\sum_{r=1}^{s_{1}} u_{r}^{g} y_{r}^{L g \max }-\sum_{p=1}^{s_{2}} u_{p}^{b} y_{p}^{U b \max }}{\sum_{i=1}^{m} v_{i} x_{i}^{U \min }}, \frac{\sum_{r=1}^{s_{1}} u_{r}^{g} y_{r}^{M g \max }-\sum_{p=1}^{s_{2}} u_{p}^{b} y_{p}^{M b \max }}{\sum_{i=1}^{m} v_{i} x_{i}^{M \min }}, \frac{\sum_{r=1}^{s_{1}} u_{r}^{g} y_{r}^{U g \max }-\sum_{p=1}^{s_{2}} u_{p}^{b} y_{p}^{L b \max }}{\sum_{i=1}^{m} v_{i} x_{i}^{M \min }}\right] \\
& \text { s.t. } \tilde{\theta}_{j}=\left[\theta_{j}^{L}, \theta_{j}^{M}, \theta_{j}^{U}\right] \\
& =\left[\frac{\sum_{r=1}^{s_{1}} u_{r}^{g} y_{r j}^{L g}-\sum_{p=1}^{s_{2}} u_{p}^{b} y_{p j}^{U b}}{\sum_{i=1}^{m} v_{i} x_{i j}^{U}}, \frac{\sum_{r=1}^{s_{1}} u_{r}^{g} y_{r j}^{M g}-\sum_{p=1}^{s_{2}} u_{p}^{b} y_{p j}^{M b}}{\sum_{i=1}^{m} v_{i} x_{i j}^{M}}, \frac{\sum_{r=1}^{s_{1}} u_{r}^{g} y_{r j}^{U g}-\sum_{p=1}^{s_{2}} u_{p}^{b} y_{p j}^{L b}}{\sum_{i=1}^{m} v_{i} x_{i j}^{L}}\right] \leq 1, \quad j=1, \ldots, n, \\
& {\left[\sum_{r=1}^{s_{1}} u_{r}^{g} y_{r j}^{L g}-\sum_{p=1}^{s_{2}} u_{p}^{b} y_{p j}^{U b}, \sum_{r=1}^{s_{1}} u_{r}^{g} y_{r j}^{M g}-\sum_{p=1}^{s_{2}} u_{p}^{b} y_{p j}^{M b}, \sum_{r=1}^{s_{1}} u_{r}^{g} y_{r j}^{U g}-\sum_{p=1}^{s_{2}} u_{p}^{b} y_{p j}^{L b}\right] \geq 0, j=1, \ldots, n,} \\
& {\left[\sum_{r=1}^{s_{1}} u_{r}^{g} y_{r}^{L g \max }-\sum_{p=1}^{s_{2}} u_{p}^{b} y_{p}^{U b \max }, \sum_{r=1}^{s_{1}} u_{r}^{g} y_{r}^{M g \max }-\sum_{p=1}^{s_{2}} u_{p}^{b} y_{p}^{M b \max }, \sum_{r=1}^{s_{1}} u_{r}^{g} y_{r}^{U g \max }-\sum_{p=1}^{s_{2}} u_{p}^{b} y_{p}^{L b \max }\right] \geq 0,}
\end{aligned}
$$

$u_{r}^{g} \geq \varepsilon \forall r, \quad u_{p}^{b} \geq \varepsilon \forall p, \quad v_{i} \geq \varepsilon \forall i$. 


$$
\begin{aligned}
& \tilde{\theta}_{I}^{*}=\max \tilde{\theta}_{I} \approx\left[\theta_{I}^{L}, \theta_{I}^{M}, \theta_{I}^{U}\right] \\
& =\left[\frac{\sum_{r=1}^{s_{1}} u_{r}^{g} y_{r}^{L g \max }-\sum_{p=1}^{s_{2}} u_{p}^{b} y_{p}^{U b \max }}{\sum_{i=1}^{m} v_{i} x_{i}^{U \min }}, \frac{\sum_{r=1}^{s_{1}} u_{r}^{g} y_{r}^{M g \max }-\sum_{p=1}^{s_{2}} u_{p}^{b} y_{p}^{M b \max }}{\sum_{i=1}^{m} v_{i} x_{i}^{M \min }}, \frac{\sum_{r=1}^{s_{1}} u_{r}^{g} y_{r}^{U g \max }-\sum_{p=1}^{s_{2}} u_{p}^{b} y_{p}^{L b \max }}{\sum_{i=1}^{m} v_{i} x_{i}^{L \min }}\right] \\
& \text { s.t. } \frac{\sum_{r=1}^{s_{1}} u_{r}^{g} y_{r j}^{U g}-\sum_{p=1}^{s_{2}} u_{p}^{b} y_{p j}^{L b}}{\sum_{i=1}^{m} v_{i} x_{i j}^{L}} \leq 1, \quad j=1, \ldots, n, \\
& \sum_{r=1}^{s_{1}} u_{r}^{g} y_{r j}^{L g}-\sum_{p=1}^{s_{2}} u_{p}^{b} y_{p j}^{U b} \geq 0, j=1, \ldots, n, \\
& \sum_{r=1}^{s_{1}} u_{r}^{g} y_{r}^{L g \max }-\sum_{p=1}^{s_{2}} u_{p}^{b} y_{p}^{U b \max } \geq 0, \\
& u_{r}^{g} \geq \varepsilon \forall r, \quad u_{p}^{b} \geq \varepsilon \forall p, \quad v_{i} \geq \varepsilon \forall i .
\end{aligned}
$$

Note that model (29) is still a fuzzy model with one fuzzy variable in the objective function. We propose a new method to solve model (29) using the conversion of this model into a multi-objective linear programming (MOLP) problem. Thus, maximizing the fuzzy efficiency $\tilde{\theta}_{I}$, which has three components $\left[\theta_{I}^{L}, \theta_{I}^{M}, \theta_{I}^{U}\right]$, leads to the following MOLP model with three objective functions for maximizing these three variables simultaneously:

$\theta_{I}^{* L}=\max \theta_{I}^{L}=\frac{\sum_{r=1}^{s_{1}} u_{r}^{g} y_{r}^{L g \max }-\sum_{p=1}^{s_{2}} u_{p}^{b} y_{p}^{U b \max }}{\sum_{i=1}^{m} v_{i} x_{i}^{U \min }}$

$\theta_{I}^{* M}=\max \theta_{I}^{M}=\frac{\sum_{r=1}^{s_{1}} u_{r}^{g} y_{r}^{M g \max }-\sum_{p=1}^{s_{2}} u_{p}^{b} y_{p}^{M b \max }}{\sum_{i=1}^{m} v_{i} x_{i}^{M \min }}$

$\theta_{I}^{* U}=\max \theta_{I}^{U}=\frac{\sum_{r=1}^{s_{1}} u_{r}^{g} y_{r}^{U g \max }-\sum_{p=1}^{s_{2}} u_{p}^{b} y_{p}^{L b \max }}{\sum_{i=1}^{m} v_{i} x_{i}^{L \min }}$

s.t. Constraints of model (29).

Now, to find the fuzzy relative efficiency of FIDMU, we use a lexicographic approach for solving MOLP model (30). Therefore, the following model is solved at first:

$\theta_{I}^{* L}=\max \theta_{I}^{L}=\frac{\sum_{r=1}^{s_{1}} u_{r}^{g} y_{r}^{L g \max }-\sum_{p=1}^{s_{2}} u_{p}^{b} y_{p}^{U b \max }}{\sum_{i=1}^{m} v_{i} x_{i}^{U \min }}$

s.t. Constraints of model (29).

By using Charnes-Cooper transformation, the model (31) can be transformed into a linear programming problem.

The second step in the lexicographic optimization of model (30) consists in solving the following model:
$\theta_{I}^{* M}=\max \theta_{I}^{M}=\frac{\sum_{r=1}^{s_{1}} u_{r}^{g} y_{r}^{M g \max }-\sum_{p=1}^{s_{2}} u_{p}^{b} y_{p}^{M b \max }}{\sum_{i=1}^{m} v_{i} x_{i}^{M \min }}$

s.t. $\theta_{I}^{L}=\theta_{I}^{* L}$,

Constraints of model (29).

Clearly, the constraint $\sum_{r=1}^{s_{1}} u_{r}^{g} y_{r}^{L g \max }-$ $\sum_{p=1}^{s_{2}} u_{p}^{b} y_{p}^{U b \max } \geq 0$ of model (29) will be redundant for model (32) and can be removed. Similarly, by using Charnes-Cooper transformation, the model (32) can be transformed into a linear programming problem.

The third step in the lexicographic optimization of model (30) consists in solving the following model:

$$
\begin{gathered}
\theta_{I}^{* U}=\max \theta_{I}^{U}=\frac{\sum_{r=1}^{s_{1}} u_{r}^{g} y_{r}^{L g \max }-\sum_{p=1}^{s_{2}} u_{p}^{b} y_{p}^{U b \max }}{\sum_{i=1}^{m} v_{i} x_{i}^{L \min }} \\
\text { s.t. } \theta_{I}^{M}=\theta_{I}^{* M} \\
\theta_{I}^{L}=\theta_{I}^{* L},
\end{gathered}
$$

Constraints of model (29).

The model (33) can be transformed into a linear programming problem by using Charnes-Cooper transformation.

The optimal values of models (31)-(33) give the fuzzy efficiency of FIDMU as $\tilde{\theta}_{I}^{*} \approx\left[\theta_{I}^{* L}, \theta_{I}^{* M}, \theta_{I}^{* U}\right]$.

It must be pointed out that the main reason to use the lexicographic approach for model (30) is to find the optimal solution of model (29). When we solve the problem of each objective function of model (30) with the constraint conditions, separately, the obtained optimal solutions of the resulting problems may or may not provide the optimal solution of model (29). In such a case, the following three separate problems are solved: 
$\theta_{I}^{* L}=\max \theta_{I}^{L}=\frac{\sum_{r=1}^{s_{1}} u_{r}^{g} y_{r}^{L g \max }-\sum_{p=1}^{s_{2}} u_{p}^{b} y_{p}^{U b \max }}{\sum_{i=1}^{m} v_{i} x_{i}^{U \min }}$

s.t. Constraints of model (29).

$\theta_{I}^{* M}=\max \theta_{I}^{M}=\frac{\sum_{r=1}^{s_{1}} u_{r}^{g} y_{r}^{M g \max }-\sum_{p=1}^{s_{2}} u_{p}^{b} y_{p}^{M b \max }}{\sum_{i=1}^{m} v_{i} x_{i}^{M \min }}$

s.t. Constraints of model (29).

$\theta_{I}^{* U}=\max \theta_{I}^{U}=\frac{\sum_{r=1}^{s_{1}} u_{r}^{g} y_{r}^{U g \max }-\sum_{p=1}^{s_{2}} u_{r}^{b} y_{p}^{L b \max }}{\sum_{i=1}^{m} v_{i} x_{i}^{L \min }}$

s.t. Constraints of model (29).

It is obvious that solving problems (A), (B) and (C) may provide different optimal solution $\left(v_{i}^{*}, u_{r}^{* g}, u_{r}^{* b}\right)$. Assume that $\left(v_{i}^{* A}, u_{r}^{* g A}, u_{r}^{* b A}\right),\left(v_{i}^{* B}, u_{r}^{* g B}, u_{r}^{* b B}\right)$ and $\left(v_{i}^{* C}, u_{r}^{* g C}, u_{r}^{* b C}\right)$ are the optimal solutions of problems (A), (B) and (C), respectively. First, there is no criterion to choose one of these optimal solutions as the optimal solution of model (29). Second, the optimal values of these problems are as follows:

$\theta_{I}^{* L}=\max \theta_{I}^{L}=\frac{\sum_{r=1}^{s_{1}} u_{r}^{* g A} y_{r}^{L g \max }-\sum_{p=1}^{s_{2}} u_{p}^{* b A} y_{p}^{U b \max }}{\sum_{i=1}^{m} v_{i}^{* A} x_{i}^{U \min }}$,

$\theta_{I}^{* M}=\max \theta_{I}^{M}$

$$
=\frac{\sum_{r=1}^{s_{1}} u_{r}^{* g B} y_{r}^{M g \max }-\sum_{p=1}^{s_{2}} u_{p}^{* b B} y_{p}^{M b \max }}{\sum_{i=1}^{m} v_{i}^{* B} x_{i}^{M \min }},
$$

$\theta_{I}^{* U}=\max \theta_{I}^{U}=\frac{\sum_{r=1}^{s_{1}} u_{r}^{* g C} y_{r}^{U g \max }-\sum_{p=1}^{s_{2}} u_{r}^{* b C} y_{p}^{L b \max }}{\sum_{i=1}^{m} v_{i}^{* C} x_{i}^{L \min }}$

Since the values of $\theta_{I}^{* L}, \theta_{I}^{* M}$ and $\theta_{I}^{* U}$ are obtained by solving three independent crisp DEA models so the restriction $\theta_{I}^{* L} \leq \theta_{I}^{* M} \leq \theta_{I}^{* U}$ may or may not be satisfied. Hence, $\tilde{\theta}_{I}^{*}=\left(\theta_{I}^{* L}, \theta_{I}^{* M}, \theta_{I}^{* U}\right)$ may or may not preserve the form of a triangular fuzzy number. However, it is obvious that the proposed lexicographic approach overcome these limitations.

\section{The worst fuzzy efficiency of FADMU}

Here, we describe a method to find the worst fuzzy efficiency of FADMU.

Similarly, the following MOLP problem is constructed to determine the worst fuzzy efficiency of FADMU:

$$
\begin{aligned}
& \tilde{\varphi}_{N}^{*}=\min \tilde{\varphi}_{N} \approx\left[\varphi_{N}^{L}, \varphi_{N}^{M}, \varphi_{N}^{U}\right] \\
& =\left[\frac{\sum_{r=1}^{s_{1}} u_{r}^{g} y_{r}^{L g \min }-\sum_{p=1}^{s_{2}} u_{p}^{b} y_{p}^{U b \min }}{\sum_{i=1}^{m} v_{i} x_{i}^{U \max }},\right.
\end{aligned}
$$

$\frac{\sum_{r=1}^{s_{1}} u_{r}^{g} y_{r}^{M g \min }-\sum_{p=1}^{s_{2}} u_{p}^{b} y_{p}^{M b \min }}{\sum_{i=1}^{m} v_{i} x_{i}^{M \max }}$

$\left.\frac{\sum_{r=1}^{s_{1}} u_{r}^{g} y_{r}^{U g \min }-\sum_{p=1}^{s_{2}} u_{p}^{b} y_{p}^{L b \min }}{\sum_{i=1}^{m} v_{i} x_{i}^{\operatorname{Lmax}}}\right]$

s.t. $\frac{\sum_{r=1}^{s_{1}} u_{r}^{g} y_{r}^{L g \max }-\sum_{p=1}^{s_{2}} u_{p}^{b} y_{p}^{U b \max }}{\sum_{i=1}^{m} v_{i} x_{i}^{U \min }} \geq \theta_{I}^{* L}$,

$\frac{\sum_{r=1}^{s_{1}} u_{r}^{g} y_{r}^{M g \max }-\sum_{p=1}^{s_{2}} u_{p}^{b} y_{p}^{M b \max }}{\sum_{i=1}^{m} v_{i} x_{i}^{M \min }} \geq \theta_{I}^{* M}$,

$\frac{\sum_{r=1}^{s_{1}} u_{r}^{g} y_{r}^{U g \max }-\sum_{p=1}^{s_{2}} u_{p}^{b} y_{p}^{L b \max }}{\sum_{i=1}^{m} v_{i} x_{i}^{L \min }} \geq \theta_{I}^{* U}$,

$\frac{\sum_{r=1}^{s_{1}} u_{r}^{g} y_{r j}^{U g}-\sum_{p=1}^{s_{2}} u_{p}^{b} y_{p j}^{L b}}{\sum_{i=1}^{m} v_{i} x_{i j}^{L}} \leq 1, \quad j=1, \ldots, n$,

$\sum_{r=1}^{s_{1}} u_{r}^{g} y_{r j}^{L g}-\sum_{p=1}^{s_{2}} u_{p}^{b} y_{p j}^{U b} \geq 0, j=1, \ldots, n$,

$\sum_{r=1}^{s_{1}} u_{r}^{g} y_{r}^{L g \min }-\sum_{p=1}^{s_{2}} u_{p}^{b} y_{p}^{U b \min } \geq 0$,

$u_{r}^{g} \geq \varepsilon \forall r, \quad u_{p}^{b} \geq \varepsilon \forall p, \quad v_{i} \geq \varepsilon \forall i$

Note that model (34) is still a fuzzy model with fuzzy coefficients in the objective function which can be formulated as the following MOLP problem:

$\varphi_{N}^{* L}=\min \varphi_{N}^{L}=\frac{\sum_{r=1}^{s_{1}} u_{r}^{g} y_{r}^{L g \min }-\sum_{p=1}^{s_{2}} u_{p}^{b} y_{p}^{U b \min }}{\sum_{i=1}^{m} v_{i} x_{i}^{U \max }}$

$\varphi_{N}^{* M}=\min \varphi_{N}^{M}=\frac{\sum_{r=1}^{s_{1}} u_{r}^{g} y_{r}^{M g \min }-\sum_{p=1}^{s_{2}} u_{p}^{b} y_{p}^{M b \min }}{\sum_{i=1}^{m} v_{i} x_{i}^{M m a x}}$

$\varphi_{N}^{* U}=\min \varphi_{N}^{U}=\frac{\sum_{r=1}^{s_{1}} u_{r}^{g} y_{r}^{U g \min }-\sum_{p=1}^{s_{2}} u_{p}^{b} y_{p}^{L b \min }}{\sum_{i=1}^{m} v_{i} x_{i}^{\operatorname{Lmax}}}$

s.t. Constraints of model (34).

According to the lexicographic approach for solving MOLP model (35), the following model is solved at first:

$\varphi_{N}^{* L}=\min \varphi_{N}^{L}=\frac{\sum_{r=1}^{s_{1}} u_{r}^{g} y_{r}^{L g \min }-\sum_{p=1}^{s_{2}} u_{p}^{b} y_{p}^{U b \min }}{\sum_{i=1}^{m} v_{i} x_{i}^{U \max }}$

s.t. Constraints of model (34).

By using Charnes-Cooper transformation, the model (36) can be transformed into a linear programming problem.

The second step in the lexicographic optimization of model (36) consists in solving the following model: 
$\varphi_{N}^{* M}=\min \varphi_{N}^{M}=\frac{\sum_{r=1}^{s_{1}} u_{r}^{g} y_{r}^{M g \min }-\sum_{p=1}^{s_{2}} u_{p}^{b} y_{p}^{M b \min }}{\sum_{i=1}^{m} v_{i} x_{i}^{\text {Mmax }}}$

s.t. $\varphi_{N}^{L}=\varphi_{N}^{* L}$

Constraints of model (34).

Clearly, the constraint $\sum_{r=1}^{s_{1}} u_{r}^{g} y_{r}^{L g \min }-\sum_{p=1}^{s_{2}} u_{p}^{b} y_{p}^{U b \min }$ $\geq 0$ of model (34) will be redundant for model (37) and can be removed. Similarly, by using Charnes-Cooper transformation, the model (37) can be transformed into a linear programming problem.

The third step in the lexicographic optimization of model (34) consists in solving the following model:

$\tilde{\varphi}_{N}^{* U}=\min \varphi_{N}^{U}=\frac{\sum_{r=1}^{s_{1}} u_{r}^{g} y_{r}^{U g \min }-\sum_{p=1}^{s_{2}} u_{p}^{b} y_{p}^{L b \min }}{\sum_{i=1}^{m} v_{i} x_{i}^{\text {Lmax }}}$

s.t. $\varphi_{N}^{M}=\varphi_{N}^{* M}$,

$\varphi_{N}^{L}=\varphi_{N}^{* L}$,

Constraints of model (34).

The model (38) can be transformed into a linear programming problem by using Charnes-Cooper transformation.

The optimal values of models (36)-(38) give the worst fuzzy efficiency of FADMU as $\tilde{\varphi}_{N}^{*} \approx\left[\varphi_{N}^{* L}, \varphi_{N}^{* M}, \varphi_{N}^{* U}\right]$.

It is obvious that every feasible solution of model (36) is a feasible solution of model (31). This means that $\varphi_{N}^{* L} \leq \theta_{I}^{* L}$. Similarly, we can conclude that $\varphi_{N}^{* M} \leq \theta_{I}^{* M}$ and $\varphi_{N}^{* U} \leq \theta_{I}^{* U}$. Therefore, the following statement can be made:

$$
\begin{aligned}
& \tilde{\theta}_{o}^{*}=\left[\theta_{o}^{* L}, \theta_{o}^{* M}, \theta_{o}^{* U}\right]=\max \left[\theta_{o}^{L}, \theta_{o}^{M}, \theta_{o}^{U}\right] \\
& =\left[\frac{\sum_{r=1}^{s_{1}} u_{r}^{g} y_{r o}^{L g}-\sum_{p=1}^{s_{2}} u_{p}^{b} y_{p o}^{U b}}{\sum_{i=1}^{m} v_{i} x_{i o}^{U}}, \frac{\sum_{r=1}^{s_{1}} u_{r}^{g} y_{r o}^{M g}-\sum_{p=1}^{s_{2}} u_{p}^{b} y_{p o}^{M b}}{\sum_{i=1}^{m} v_{i} x_{i o}^{M}}, \frac{\sum_{r=1}^{s_{1}} u_{r}^{g} y_{r o}^{U g}-\sum_{p=1}^{s_{2}} u_{p}^{b} y_{p o}^{L b}}{\sum_{i=1}^{m} v_{i} x_{i o}^{L}}\right] \\
& \text { s.t. }\left[\frac{\sum_{r=1}^{s_{1}} u_{r}^{g} y_{r}^{L g \max }-\sum_{p=1}^{s_{2}} u_{p}^{b} y_{p}^{U b \max }}{\sum_{i=1}^{m} v_{i} x_{i}^{U \min }}, \frac{\sum_{r=1}^{s_{1}} u_{r}^{g} y_{r}^{M g \max }-\sum_{p=1}^{s_{2}} u_{p}^{b} y_{p}^{M b \max }}{\sum_{i=1}^{m} v_{i} x_{i}^{M \min }}, \frac{\sum_{r=1}^{s_{1}} u_{r}^{g} y_{r}^{U g \max }-\sum_{p=1}^{s_{2}} u_{p}^{b} y_{p}^{L b \max }}{\sum_{i=1}^{m} v_{i} x_{i}^{L \min }}\right] \\
& =\left[\theta_{I}^{* L}, \theta_{I}^{* M}, \theta_{I}^{* U}\right] \\
& {\left[\frac{\sum_{r=1}^{s_{1}} u_{r}^{g} y_{r j}^{L g}-\sum_{p=1}^{s_{2}} u_{p}^{b} y_{p j}^{U b}}{\sum_{i=1}^{m} v_{i} x_{i j}^{U}}, \frac{\sum_{r=1}^{s_{1}} u_{r}^{g} y_{r j}^{M g}-\sum_{p=1}^{s_{2}} u_{p}^{b} y_{p j}^{M b}}{\sum_{i=1}^{m} v_{i} x_{i j}^{M}}, \frac{\sum_{r=1}^{s_{1}} u_{r}^{g} y_{r j}^{U g}-\sum_{p=1}^{s_{2}} u_{p}^{b} y_{p j}^{L b}}{\sum_{i=1}^{m} v_{i} x_{i o}^{L}}\right] \leq 1, j=1, \ldots, n,} \\
& {\left[\sum_{r=1}^{s_{1}} u_{r}^{g} y_{r j}^{L g}-\sum_{p=1}^{s_{2}} u_{p}^{b} y_{p j}^{U b}, \sum_{r=1}^{s_{1}} u_{r}^{g} y_{r j}^{M g}-\sum_{p=1}^{s_{2}} u_{p}^{b} y_{p j}^{M b}, \sum_{r=1}^{s_{1}} u_{r}^{g} y_{r j}^{U g}-\sum_{p=1}^{s_{2}} u_{p}^{b} y_{p j}^{L b}\right] \geq 0, j=1, \ldots, n,}
\end{aligned}
$$$$
u_{r}^{g} \geq \varepsilon \forall r, \quad u_{p}^{b} \geq \varepsilon \forall p, \quad v_{i} \geq \varepsilon \forall i .
$$

Proposition 1 The worst fuzzy efficiency of FADMU is less or equal than the best fuzzy efficiency of the FIDMU, i.e., $\tilde{\varphi}_{N}^{*} \approx\left[\varphi_{N}^{* L}, \varphi_{N}^{* M}, \varphi_{N}^{* U}\right] \preceq \tilde{\theta}_{I}^{*} \approx\left[\theta_{I}^{* L}, \theta_{I}^{* M}, \theta_{I}^{* U}\right]$.

\section{The best fuzzy efficiency of DMU}

Now, we compute the best fuzzy relative efficiency of each DMU using the best fuzzy efficiency of FIDMU $\left(\tilde{\theta}_{I}^{*}\right)$. The relative efficiency of each DMU using the optimum fuzzy efficiency of the FIDMU:

$\tilde{\theta}_{o}^{*}=\max \frac{\sum_{r=1}^{s_{1}} u_{r}^{g} \tilde{y}_{r o}^{g}-\sum_{p=1}^{s_{2}} u_{p}^{b} \tilde{y}_{p o}^{b}}{\sum_{i=1}^{m} v_{i} \tilde{x}_{i o}}$
s.t. $\frac{\sum_{r=1}^{s_{1}} u_{r}^{g} \tilde{y}_{r}^{g \max }-\sum_{p=1}^{s_{2}} u_{p}^{b} \tilde{y}_{p}^{b \max }}{\sum_{i=1}^{m} v_{i} \tilde{x}_{i}^{\min }}=\tilde{\theta}_{I}^{*}$
$\tilde{\theta}_{j}=\frac{\sum_{r=1}^{s_{1}} u_{r}^{g} \tilde{y}_{r j}^{g}-\sum_{p=1}^{s_{2}} u_{p}^{b} \tilde{y}_{p j}^{b}}{\sum_{i=1}^{m} v_{i} \tilde{x}_{i j}} \leq 1, j=1, \ldots, n$,

$\sum_{r=1}^{s_{1}} u_{r}^{g} \tilde{y}_{r j}^{g}-\sum_{p=1}^{s_{2}} u_{p}^{b} \tilde{y}_{p j}^{b} \geq 0, j=1, \ldots, n$,

$u_{r}^{g} \geq \varepsilon \forall r, \quad u_{p}^{b} \geq \varepsilon \forall p, \quad v_{i} \geq \varepsilon \forall i$.

The purpose of fuzzy model (39) is to maximize the fuzzy efficiency of $\mathrm{DMU}_{o}(o=1,2, \ldots, n)$ while the optimal fuzzy efficiency of the FIDMU is fixed by the first constraint in the fuzzy model (3) is reformulated as follows: following fuzzy model is used to determine the best fuzzy fuzzy model (39). By the use of triangular fuzzy numbers,

$u_{r} \geq \varepsilon \forall r, \quad u_{p} \geq \varepsilon \forall p, \quad v_{i} \geq \varepsilon \forall i$ 
The first constraint of model (40) is rewritten as follows:

$\frac{\sum_{r=1}^{s_{1}} u_{r}^{g} y_{r}^{L g \max }-\sum_{p=1}^{s_{2}} u_{p}^{b} y_{p}^{U b \max }}{\sum_{i=1}^{m} v_{i} x_{i}^{U \min }}=\theta_{I}^{* L}$,

$\frac{\sum_{r=1}^{s_{1}} u_{r}^{g} y_{r}^{M g \max }-\sum_{p=1}^{s_{2}} u_{p}^{b} y_{p}^{M b \max }}{\sum_{i=1}^{m} v_{i} x_{i}^{M \min }}=\theta_{I}^{* M}$,

$\frac{\sum_{r=1}^{s_{1}} u_{r}^{g} y_{r}^{U g \max }-\sum_{p=1}^{s_{2}} u_{p}^{b} y_{p}^{L b \max }}{\sum_{i=1}^{m} v_{i} x_{i}^{L \min }}=\theta_{I}^{* U}$.

The second constraint set of model (40) is simplified to the following constraint:

$\frac{\sum_{r=1}^{s_{1}} u_{r}^{g} y_{r j}^{U g}-\sum_{p=1}^{s_{2}} u_{p}^{b} y_{p j}^{L b}}{\sum_{i=1}^{m} v_{i} x_{i o}^{L}} \leq 1, \quad j=1, \ldots, n$,

The second constraint of model (40) is simplified to the following constraint:

$\sum_{r=1}^{s_{1}} u_{r}^{g} y_{r j}^{L g}-\sum_{p=1}^{s_{2}} u_{p}^{b} y_{p j}^{U b} \geq 0, \quad j=1, \ldots, n$,

Now, regarding Eqs. (41), (42) and (43), we can rewrite model (40) as follows:

$$
\begin{aligned}
& \tilde{\theta}_{o}^{*}=\left[\theta_{o}^{* L}, \theta_{o}^{* M}, \theta_{o}^{* U}\right]=\max \left[\theta_{o}^{L}, \theta_{o}^{M}, \theta_{o}^{U}\right] \\
& =\left[\frac{\sum_{r=1}^{s_{1}} u_{r}^{g} y_{r o}^{L g}-\sum_{p=1}^{s_{2}} u_{p}^{b} y_{p o}^{U b}}{\sum_{i=1}^{m} v_{i} x_{i o}^{U}},\right. \\
& \quad \frac{\sum_{r=1}^{s_{1}} u_{r}^{g} y_{r o}^{M g}-\sum_{p=1}^{s_{2}} u_{p}^{b} y_{p o}^{M b}}{\sum_{i=1}^{m} v_{i} x_{i o}^{M}}, \\
& \left.\quad \frac{\sum_{r=1}^{s_{1}} u_{r}^{g} y_{r o}^{U g}-\sum_{p=1}^{s_{2}} u_{p}^{b} y_{p o}^{L b}}{\sum_{i=1}^{m} v_{i} x_{i o}^{L}}\right] \\
& \text { s.t. } \sum_{r=1}^{s_{1}} u_{r}^{g} y_{r}^{L g \max } \\
& \quad-\sum_{p=1}^{s_{2}} u_{p}^{b} y_{p}^{U b \max }-\theta_{I}^{* L} \sum_{i=1}^{m} v_{i} x_{i}^{U \min }=0, \\
& \sum_{r=1}^{s_{1}} u_{r}^{g} y_{r}^{M g \max }-\sum_{p=1}^{s_{2}} u_{p}^{b} y_{p}^{M b \max }-\theta_{I}^{* M} \sum_{i=1}^{m} v_{i} x_{i}^{M \min }=0, \\
& \sum_{r=1}^{s_{1}} u_{r}^{g} y_{r}^{U g \max }-\sum_{p=1}^{s_{2}} u_{p}^{b} y_{p}^{L b \max }-\theta_{I}^{* U} \sum_{i=1}^{m} v_{i} x_{i}^{L \min }=0, \\
& \sum_{r=1}^{s_{1}} u_{r}^{g} y_{r j}^{U g}-\sum_{p=1}^{s_{2}} u_{p}^{b} y_{p j}^{L b}-\sum_{i=1}^{m} v_{i} x_{i j}^{L} \leq 0, \\
& \sum_{r}^{s_{1}} u_{r}^{g} y_{r j}^{L g}-\sum_{p=1}^{s_{2}} u_{p}^{b} y_{p j}^{U b} \geq 0, \quad j=1, \ldots, n, \\
& u_{r}^{g} \geq \varepsilon \forall r, \quad u_{p}^{b} \geq \varepsilon \forall p, \quad v_{i} \geq \varepsilon \forall i .
\end{aligned}
$$

The first, second and third steps in the lexicographic optimization of model (44) involve solving, successively, the following three models:

$\theta_{o}^{* L}=\max \theta_{o}^{L}=\frac{\sum_{r=1}^{s_{1}} u_{r}^{g} y_{r o}^{L g}-\sum_{p=1}^{s_{2}} u_{p}^{b} y_{p o}^{U b}}{\sum_{i=1}^{m} v_{i} x_{i o}^{U}}$

s.t. Constraints of model (44).

$\theta_{o}^{* M}=\max \theta_{o}^{M}=\frac{\sum_{r=1}^{s_{1}} u_{r}^{g} y_{r o}^{M g}-\sum_{p=1}^{s_{2}} u_{p}^{b} y_{p o}^{M b}}{\sum_{i=1}^{m} v_{i} x_{i o}^{M}}$

s.t. $\theta_{o}^{L}=\theta_{o}^{* L}$,

Constraints of model (44).

$\theta_{o}^{* U}=\max \theta_{o}^{U}=\frac{\sum_{r=1}^{s_{1}} u_{r}^{g} y_{r o}^{U g}-\sum_{p=1}^{s_{2}} u_{p}^{b} y_{p o}^{L b}}{\sum_{i=1}^{m} v_{i} x_{i o}^{L}}$

s.t. $\theta_{o}^{M}=\theta_{o}^{* M}$,

$\theta_{o}^{L}=\theta_{o}^{* L}$,

Constraints of model (44).

It should be noted that every feasible solution of model (46) is also a feasible solution of model (31). This means that $\theta_{o}^{* L} \leq \theta_{I}^{* L}(0=1,2, \ldots, n)$. Similarly, $\theta_{o}^{* M} \leq \theta_{I}^{* M}(0=$ $1,2, \ldots, n)$ and $\theta_{o}^{* U} \leq \theta_{I}^{* U}(0=1,2, \ldots, n)$. Therefore, the following statement can be made:

Proposition 2 The best fuzzy efficiency of every DMU under evaluation is less or equal than the best fuzzy efficiency of the FIDMU, i.e., $\tilde{\theta}_{j}^{*} \approx\left[\theta_{j}^{* L}, \theta_{j}^{* M}, \theta_{j}^{* U}\right] \underline{\theta_{I}^{*}} \approx\left[\theta_{I}^{* L}, \theta_{I}^{* M}\right.$, $\left.\theta_{I}^{* U}\right],(j=1,2, \ldots, n)$.

\section{The worst fuzzy efficiency of DMU}

Now, we compute the worst fuzzy relative efficiency of each DMU using the worst fuzzy efficiency of FADMU $\left(\tilde{\varphi}_{N}^{*}\right)$. The following fuzzy model is used to determine the worst fuzzy relative efficiency of each DMU using the optimum fuzzy efficiency of the FADMU:

$$
\begin{aligned}
& \tilde{\varphi}_{o}^{*}=\min \frac{\sum_{r=1}^{s_{1}} u_{r}^{g} \tilde{y}_{r o}^{g}-\sum_{p=1}^{s_{2}} u_{p}^{b} \tilde{y}_{p o}^{b}}{\sum_{i=1}^{m} v_{i} \tilde{x}_{i o}} \\
& \text { s.t. } \frac{\sum_{r=1}^{s_{1}} u_{r}^{g} \tilde{y}_{r}^{g \min }-\sum_{p=1}^{s_{2}} u_{p}^{b} \tilde{y}_{p}^{b \min }}{\sum_{i=1}^{m} v_{i} \tilde{x}_{i}^{\max }}=\tilde{\varphi}_{N}^{*} \\
& \tilde{\varphi}_{j}=\frac{\sum_{r=1}^{s_{1}} u_{r}^{g} \tilde{y}_{r j}^{g}-\sum_{p=1}^{s_{2}} u_{p}^{b} \tilde{y}_{p j}^{b}}{\sum_{i=1}^{m} v_{i} \tilde{x}_{i j}} \leq 1, j=1, \ldots, n, \\
& \sum_{r=1}^{s_{1}} u_{r}^{g} \tilde{y}_{r j}^{g}-\sum_{p=1}^{s_{2}} u_{p}^{b} \tilde{y}_{p j}^{b} \geq 0, j=1, \ldots, n, \\
& u_{r}^{g} \geq \varepsilon \forall r, \quad u_{p}^{b} \geq \varepsilon \forall p, \quad v_{i} \geq \varepsilon \forall i .
\end{aligned}
$$


The purpose of fuzzy model (48) is to minimize the fuzzy efficiency of $\operatorname{DMU}_{o}(o=1,2, \ldots, n)$ while the optimal fuzzy efficiency of the FADMU is fixed by the first constraint in fuzzy model (48). By the use of triangular fuzzy numbers, the fuzzy model (3) is reformulated as follows:
The first, second and third steps in the lexicographic optimization of model (50) involve solving, successively, the following three models:

$$
\begin{aligned}
& \tilde{\varphi}_{o}^{*}=\min \left[\varphi_{o}^{L}, \varphi_{o}^{M}, \varphi_{o}^{U}\right] \\
& =\left[\frac{\sum_{r=1}^{s_{1}} u_{r}^{g} y_{r o}^{L g}-\sum_{p=1}^{s_{2}} u_{p}^{b} y_{p o}^{U b}}{\sum_{i=1}^{m} v_{i} x_{i o}^{U}}, \frac{\sum_{r=1}^{s_{1}} u_{r}^{g} y_{r o}^{M g}-\sum_{p=1}^{s_{2}} u_{p}^{b} y_{p o}^{M b}}{\sum_{i=1}^{m} v_{i} x_{i o}^{M}}, \frac{\sum_{r=1}^{s_{1}} u_{r}^{g} y_{r o}^{U g}-\sum_{p=1}^{s_{2}} u_{p}^{b} y_{p o}^{L b}}{\sum_{i=1}^{m} v_{i} x_{i o}^{L}}\right] \\
& \text { s.t. }\left[\frac{\sum_{r=1}^{s_{1}} u_{r}^{g} y_{r}^{L g \min }-\sum_{p=1}^{s_{2}} u_{p}^{b} y_{p}^{U b \min }}{\sum_{i=1}^{m} v_{i} x_{i}^{U m a x}}, \frac{\sum_{r=1}^{s_{1}} u_{r}^{g} y_{r}^{M g \min }-\sum_{p=1}^{s_{2}} u_{p}^{b} y_{p}^{M b \min }}{\sum_{i=1}^{m} v_{i} x_{i}^{M m a x}}, \frac{\sum_{r=1}^{s_{1}} u_{r}^{g} y_{r}^{U g \min }-\sum_{p=1}^{s_{2}} u_{p}^{b} y_{p}^{L b \min }}{\sum_{i=1}^{m} v_{i} x_{i}^{L m a x}}\right] \\
& =\left[\varphi_{N}^{* L}, \varphi_{N}^{* M}, \varphi_{N}^{* U}\right] \\
& {\left[\frac{\sum_{r=1}^{s_{1}} u_{r}^{g} y_{r j}^{L g}-\sum_{p=1}^{s_{2}} u_{p}^{b} y_{p j}^{U b}}{\sum_{i=1}^{m} v_{i} x_{i j}^{U}}, \frac{\sum_{r=1}^{s_{1}} u_{r}^{g} y_{r j}^{M g}-\sum_{p=1}^{s_{2}} u_{p}^{b} y_{p j}^{M b}}{\sum_{i=1}^{m} v_{i} x_{i j}^{M}}, \frac{\sum_{r=1}^{s_{1}} u_{r}^{g} y_{r j}^{U g}-\sum_{p=1}^{s_{2}} u_{p}^{b} y_{p j}^{L b}}{\sum_{i=1}^{m} v_{i} x_{i j}^{L}}\right] \leq 1, j=1, \ldots, n,} \\
& {\left[\sum_{r=1}^{s_{1}} u_{r}^{g} y_{r j}^{L g}-\sum_{p=1}^{s_{2}} u_{p}^{b} y_{p j}^{U b}, \sum_{r=1}^{s_{1}} u_{r}^{g} y_{r j}^{M g}-\sum_{p=1}^{s_{2}} u_{p}^{b} y_{p j}^{M b}, \sum_{r=1}^{s_{1}} u_{r}^{g} y_{r j}^{U g}-\sum_{p=1}^{s_{2}} u_{p}^{b} y_{p j}^{L b}\right] \geq 0, j=1, \ldots, n,} \\
& u_{r}^{g} \geq \varepsilon \forall r, \quad u_{p}^{b} \geq \varepsilon \forall p, \quad v_{i} \geq \varepsilon \forall i .
\end{aligned}
$$

Similar to previous discussions, the fuzzy DEA model (49) is reformulated as follows:

$$
\begin{aligned}
\tilde{\varphi}_{o}^{*}=\min \left[\varphi_{o}^{L}, \varphi_{o}^{M}, \varphi_{o}^{U}\right] & {\left[\frac{\sum_{r=1}^{s_{1}} u_{r}^{g} y_{r o}^{L g}-\sum_{p=1}^{s_{2}} u_{p}^{b} y_{p o}^{U b}}{\sum_{i=1}^{m} v_{i} x_{i o}^{U}},\right.} \\
& \frac{\sum_{r=1}^{s_{1}} u_{r}^{g} y_{r o}^{M g}-\sum_{p=1}^{s_{2}} u_{p}^{b} y_{p o}^{M b}}{\sum_{i=1}^{m} v_{i} x_{i o}^{M}}, \\
& \left.\frac{\sum_{r=1}^{s_{1}} u_{r}^{g} y_{r o}^{U g}-\sum_{p=1}^{s_{2}} u_{p}^{b} y_{p o}^{L b}}{\sum_{i=1}^{m} v_{i} x_{i o}^{L}}\right]
\end{aligned}
$$

s.t. $\sum_{r=1}^{s_{1}} u_{r}^{g} y_{r}^{L g \min }-\sum_{p=1}^{s_{2}} u_{p}^{b} y_{p}^{U b \min }-\varphi_{N}^{* L} \sum_{i=1}^{m} v_{i} x_{i}^{U m a x}=0$,

$\sum_{r=1}^{s_{1}} u_{r}^{g} y_{r}^{M g \min }-\sum_{p=1}^{s_{2}} u_{p}^{b} y_{p}^{M b \min }-\varphi_{N}^{* M} \sum_{i=1}^{m} v_{i} x_{i}^{M \max }=0$,

$\sum_{r=1}^{s_{1}} u_{r}^{g} y_{r}^{U g \min }-\sum_{p=1}^{s_{2}} u_{p}^{b} y_{p}^{L b \min }-\varphi_{N}^{* U} \sum_{i=1}^{m} v_{i} x_{i}^{L m a x}=0$,

$\sum_{r=1}^{s_{1}} u_{r}^{g} y_{r j}^{U g}-\sum_{p=1}^{s_{2}} u_{p}^{b} y_{p j}^{L b}-\sum_{i=1}^{m} v_{i} x_{i j}^{L} \leq 0, \quad j=1, \ldots, n$,

$\sum_{r=1}^{s_{1}} u_{r}^{g} y_{r j}^{L g}-\sum_{p=1}^{s_{2}} u_{p}^{b} y_{p j}^{U b} \geq 0, \quad j=1, \ldots, n$,

$u_{r}^{g} \geq \varepsilon \forall r, \quad u_{p}^{b} \geq \varepsilon \forall p, \quad v_{i} \geq \varepsilon \forall i$. $\varphi_{o}^{* L}=\min \frac{\sum_{r=1}^{s_{1}} u_{r}^{g} y_{r o}^{L g}-\sum_{p=1}^{s_{2}} u_{p}^{b} y_{p o}^{U b}}{\sum_{i=1}^{m} v_{i} x_{i o}^{U}}$

s.t. Constraints of model (50).

$\varphi_{o}^{* M}=\min \frac{\sum_{r=1}^{s_{1}} u_{r}^{g} y_{r o}^{M g}-\sum_{p=1}^{s_{2}} u_{p}^{b} y_{p o}^{M b}}{\sum_{i=1}^{m} v_{i} x_{i o}^{M}}$

s.t. $\varphi_{o}^{L}=\varphi_{o}^{* L}$

Constraints of model (50).

$\varphi_{o}^{* U}=\min \frac{\sum_{r=1}^{s_{1}} u_{r}^{g} y_{r o}^{U g}-\sum_{p=1}^{s_{2}} u_{p}^{b} y_{p o}^{L b}}{\sum_{i=1}^{m} v_{i} x_{i o}^{L}}$

s.t. $\varphi_{o}^{M}=\varphi_{o}^{* M}$,

$\varphi_{o}^{L}=\varphi_{o}^{* L}$,

Constraints of model (50).

The optimal values of models (51)-(53) yields the worst fuzzy efficiency of $\mathrm{DMU}_{o}(0=1,2, \ldots, n)$. It should be noted that every feasible solution of model (51) is also a feasible solution of model (36). This means that $\varphi_{o}^{* L} \leq \varphi_{N}^{* L}(0=$ $1,2, \ldots, n)$. Similarly, $\varphi_{o}^{* M} \leq \varphi_{N}^{* M}(0=1,2, \ldots, n)$ and $\varphi_{o}^{* U} \leq \varphi_{N}^{* U}(0=1,2, \ldots, n)$. Therefore, the following statement can be made:

Proposition 3 The worst fuzzy efficiency of every DMU under evaluation is more or equal than the worst fuzzy efficiency 
of the FADMU, i.e., $\tilde{\varphi}_{j}^{*} \approx\left[\varphi_{j}^{* L}, \varphi_{j}^{* M}, \varphi_{j}^{* U}\right] \succeq \tilde{\varphi}_{N}^{*} \approx\left[\varphi_{N}^{* L}\right.$, $\left.\varphi_{N}^{* M}, \varphi_{N}^{* U}\right],(j=1,2, \ldots, n)$.

Proposition 4 The worst fuzzy efficiency of every DMU under evaluation is less or equal than the best fuzzy efficiency of the same $D M U$, i.e., $\tilde{\varphi}_{j}^{*} \approx\left[\varphi_{j}^{* L}, \varphi_{j}^{* M}, \varphi_{j}^{* U}\right] \preceq \tilde{\theta}_{j}^{*} \approx\left[\theta_{j}^{* L}\right.$, $\left.\theta_{j}^{* M}, \theta_{j}^{* U}\right],(j=1,2, \ldots, n)$.

\section{Fuzzy relative closeness to FIDMU}

Here, we describe a method to integrate the best and worst fuzzy efficiencies of FDMU in order to find a total ranking of DMUs.

In short, the best fuzzy relative efficiencies of virtual FIDMU and real fuzzy $\operatorname{DMU}_{o}(0=1,2, \ldots, n)$ are computed based on models (31)-(33) and (45)-(47), respectively. Moreover, the worst fuzzy relative efficiencies of virtual FADMU and real fuzzy $\operatorname{DMU}_{o}(o=1,2, \ldots, n)$ are computed based on models (36)-(38) and (51)-(53), respectively. The fuzzy best and worst efficiency assessments may lead to completely different results. To overcome this problem, we extend the concept of the fuzzy relative closeness to give an overall assessment of each DMU. The fuzzy relative closeness to FIDMU is defined as follows:

$\underset{o}{\mathrm{RC}}=\frac{\tilde{\varphi}_{o}^{*}-\tilde{\varphi}_{N}^{*}}{\left(\tilde{\varphi}_{o}^{*}-\tilde{\varphi}_{N}^{*}\right)+\left(\tilde{\theta}_{I}^{*}-\tilde{\theta}_{o}^{*}\right)}$

The proposed approach uses $\underset{o}{\mathrm{RC}}$ to rank $\mathrm{DMU}_{o}(0=1$, $2, \ldots, n)$. The larger $\tilde{\mathrm{RC}}$ is, the larger is the rank of $\mathrm{DMU}_{o}$.

By solving models (31)-(33), (45)-(47), (36)-(38) and (51)-(53), we can get the fuzzy relative closeness of the $n$ fuzzy DMUs according to Eq. (54), based on which the DMUs can be compared and ranked with the help of the ranking approach developed in the Sect. 3.1. This means that each $\underset{o}{R} C(o=1,2, \ldots, n)$ is considered as a fuzzy number and ranked according to Eq. (7).

Remark 3 Note that if the all data are crisp numbers, then our fuzzy DEA models are reduced to the crisp DEA models. In fact, if $x_{i j}^{L}=x_{i j}^{M}=x_{i j}^{U}=x_{i j}$ and $y_{r j}^{L}=y_{r j}^{M}=y_{r j}^{U}=y_{r j}$, then (23) is reduced to (15) and so FIDMU and FADMU are reduced to IDMU and ADMU, respectively. Consequently, models (26), (34), (39) and (48) are simplified to (16)-(19), respectively. Finally, the fuzzy relative closeness to FIDMU given in (54) is simplified to the relative closeness to IDMU given in Remark 1 . This confirms in such a case these results are consistent with the results of crisp DEA formulation.

Figure 1 sums up the proposed framework in this study using five structured successive phases.

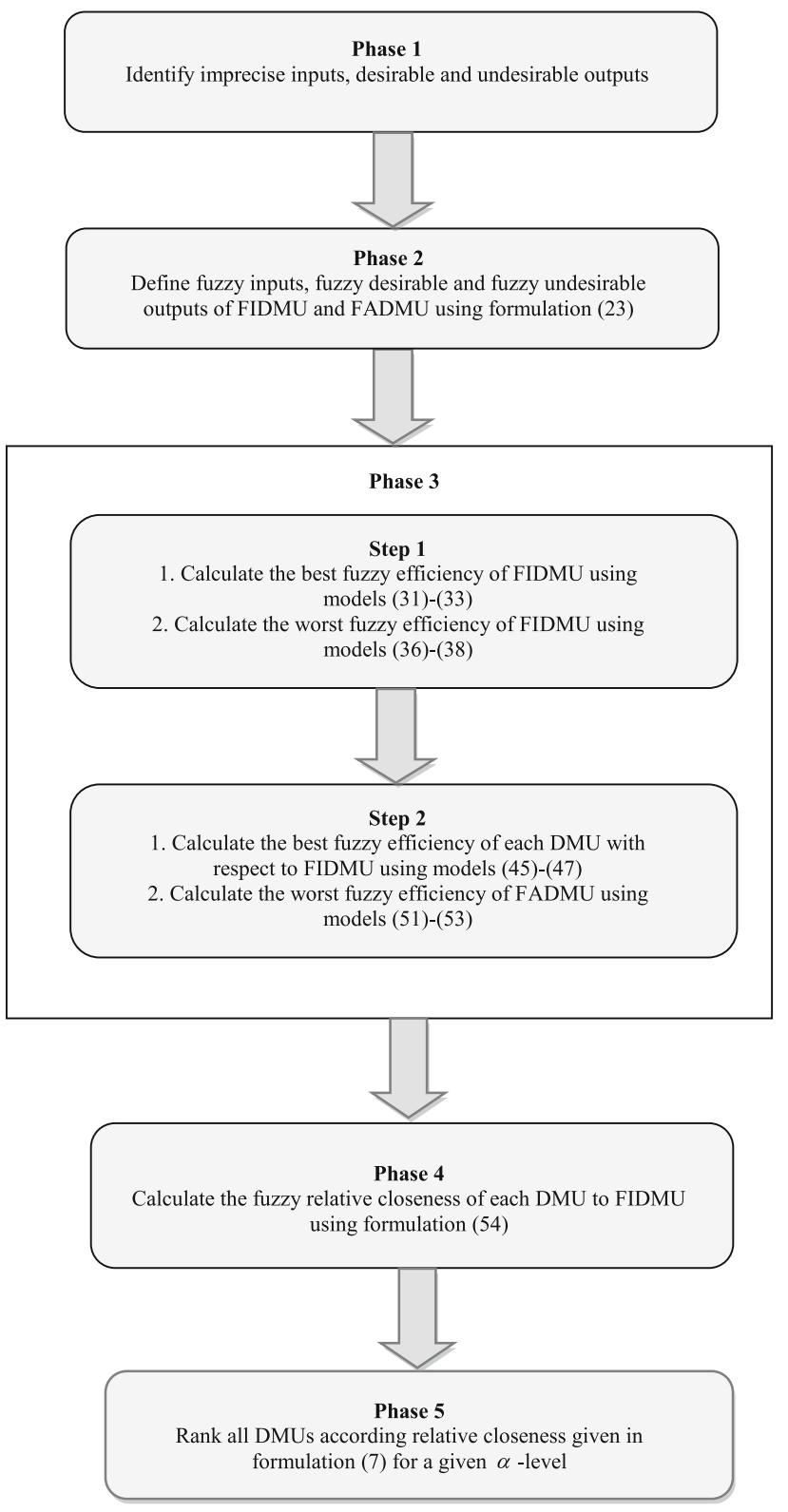

Fig. 1 The proposed algorithm

\section{Numerical example}

To illustrate the efficiency of the proposed method, we examine a numerical example. This example was originally introduced by Puri and Yadav [50]. Twelve DMUs are considered with two inputs, two desirable outputs and one undesirable output, where all the input and output data are triangular fuzzy numbers as listed in Table 2 . The virtual FIDMU and FADMU are defined in the last two rows of Table 2.

By solving models (31)-(33), the best fuzzy relative efficiency of FIADM is obtained as $\tilde{\theta}_{I}^{*}=$ $(1.7195,1.8689,2.0666)$. Moreover, by solving models 
Table 2 Fuzzy input-output data, FIDMU and FADMU

\begin{tabular}{llllll}
\hline DMU & Input 1 & Input 2 & $\begin{array}{l}\text { Output } 1 \\
\text { desirable) }\end{array}$ & $\begin{array}{l}\text { Output } 1 \\
\text { (desirable) }\end{array}$ & $\begin{array}{l}\text { Output } 1 \\
\text { (undesirable) }\end{array}$ \\
\hline 1 & $(16,20,22)$ & $(150,151,152)$ & $(95,100,102)$ & $(87,90,94)$ & $(1,1,1)$ \\
2 & $(18,19,20)$ & $(130,131,132)$ & $(149,150,151)$ & $(46,50,52)$ & $(1,2,2.5)$ \\
3 & $(23,25,28)$ & $(158,160,162)$ & $(158,160,163)$ & $(53,55,56)$ & $(1.4,2,3)$ \\
4 & $(26,27,29)$ & $(165,168,169)$ & $(177,180,181)$ & $(70,72,75)$ & $(1,1,1)$ \\
5 & $(20,22,25)$ & $(155,158,162)$ & $(90,94,98)$ & $(63,66,68)$ & $(3,5,6)$ \\
6 & $(52,55,59)$ & $(250,255,259)$ & $(222,230,235)$ & $(83,90,95)$ & $(3,4,5)$ \\
7 & $(30,33,34)$ & $(234,235,236)$ & $(210,220,225)$ & $(81,88,90)$ & $(1,3,5)$ \\
8 & $(27,31,33)$ & $(202,206,208)$ & $(151,152,155)$ & $(75,80,84)$ & $(4,5,6)$ \\
9 & $(26,30,35)$ & $(240,244,247)$ & $(188,190,193)$ & $(99,100,101)$ & $(2,5,7)$ \\
10 & $(47,50,54)$ & $(262,268,271)$ & $(246,250,252)$ & $(94,100,108)$ & $(1,3,4)$ \\
11 & $(50,53,56)$ & $(300,306,309)$ & $(255,260,264)$ & $(143,147,152)$ & $(4.5,5,5.6)$ \\
12 & $(30,38,42)$ & $(283,284,285)$ & $(246,250,254)$ & $(116,120,123)$ & $(2,3,4)$ \\
FIDMU & $(16,19,20)$ & $(130,131,132)$ & $(255,260,264)$ & $(143,147,152)$ & $(4.5,5,7)$ \\
FADMU & $(52,55,59)$ & $(300,306,309)$ & $(90,94,98)$ & $(46,50,52)$ & $(1,1,1)$ \\
\hline
\end{tabular}

Table 3 The best and worst fuzzy relative efficiency values

\begin{tabular}{lllll}
\hline DMU & $\tilde{\theta}_{j}^{*}$ & $\tilde{\varphi}_{j}^{*}$ & $\tilde{\theta}_{I}^{*}$ & $\tilde{\varphi}_{N}^{*}$ \\
\hline 1 & $(0.8240,0.8924,1.0)$ & $(0.7359,0.8172,0.9409)$ & - & - \\
2 & $(0.8667,0.9291,1.0)$ & $(0.6459,07,434,0.9464)$ & - & - \\
3 & $(0.7168,0.7975,0.8624)$ & $(0.5044,0.6645,0.7830)$ & - & - \\
4 & $(0.8632,0.9115,1.00)$ & $(0.6252,0.8272,0.8696)$ & - & - \\
5 & $(0.4811,0.5686,0.6739)$ & $(0.3011,0.3676,0.4310)$ & - & - \\
6 & $(0.5614,0.6313,0.6914)$ & $(0.4102,0.4647,0.5672)$ & - & - \\
7 & $(0.7174,0.8099,0.8977)$ & $(0.4642,0.6798,0.8788)$ & - & - \\
8 & $(0.5699,0.6261,0.7123)$ & $0.2234,0.3397,0.4864)$ & - & - \\
9 & $(0.6481,0.7348,0.8474)$ & $(0.3011,0.4950,0.8026)$ & - & - \\
10 & $(0.6588,0.7221,0.8027)$ & $(0.4998,0.6075,0.6975)$ & - & - \\
11 & $(0.6996,0.7462,0.8007)$ & $0.5091,0.5783,0.6505)$ & - & - \\
12 & $(0.8049,0.8297,0.9364)$ & $(0.6607,0.7400,0.9181)$ & - & - \\
FIDMU & - & - & - & - \\
FADMU & - & - & - & $(1.7195,1.8689,2.0666)$ \\
\end{tabular}

(36)-(38), the worst fuzzy relative efficiencies of virtual FADMU are yielded as $\tilde{\varphi}_{N}^{*}=(0.2136,0.2400,0.2623)$. We use models (45)-(47) to compute the best fuzzy relative efficiency of the real DMUs and models (51)-(53) to measure the worst fuzzy relative efficiency of the real DMUs. The resulting fuzzy efficiencies are presented in Table 3.

Now, we obtain the fuzzy relative closeness of the fuzzy DMUs according to Eq. (54) DMUs. The resulting fuzzy relative closeness is presented in Table 4.

To provide full ranking of performance for the 12 DMUs, Tables 5 and 6 show the values of $S_{\min }^{\alpha}$ and $S_{\max }^{\alpha}$ at different levels of $\alpha \in(0,1]$ calculated by Eqs. (3) and (5), respectively.

Table 7 shows the relative closeness for the twelve DMUs at different levels of $\alpha \in(0,1]$ calculated by Eq. (7). Table
8 gives the ranking results based on obtained relative closeness of DMUs. The graphical representation of the relative closeness values is shown in Fig. 2.

As can be seen from Table 7 when the value of $\alpha$ increases the relative closeness values for all DMUs increase. The relative closeness values obtained for the twelve DMUs at different levels $\alpha \in$ $\{0.1,0.2,0.3,0.4,0.5,0.6,0.7,0.8,0.9,1.0\} \quad$ illustrated in Fig. 2 which can be helpful for the sensitivity analysis of DMUs. Figures 3 and 4 show the ranking results of the DMUs according to the proposed method and the Puri and Yadav's approach [50] method, respectively. Figure 3 illustrates the extent of variations in the ranking of DMUs at different levels $\alpha \in\{0.1,0.2,0.3,0.4,0.5,0.6,0.7,0.8,0.9,1.0\}$. This figure shows the influence of an optimistic $\alpha$ on 
Table 4 Values of $\tilde{R C}_{j}$

\begin{tabular}{ll}
\hline DMU & $R \tilde{C}_{j}$ \\
\hline 1 & $(0.2404,0.3715,0.6096)$ \\
2 & $(0.1985,0.3489,0.6643)$ \\
3 & $(0.1242,0.2838,0.5180)$ \\
4 & $(0.1952,0.3802,0.5799)$ \\
5 & $(0.0216,0.0895,0.2006)$ \\
6 & $(0.0796,0.1537,0.3007)$ \\
7 & $(0.1003,0.2935,0.6498)$ \\
8 & $(0.1747,0.0744,0.2818)$ \\
9 & $(0.0194,0.1837,0.6466)$ \\
10 & $(0.1256,0.2428,0.4193)$ \\
11 & $(0.1368,0.2316,0.3748)$ \\
12 & $(0.2027,0.3249,0.5963)$ \\
\hline
\end{tabular}

Table 5 The values of $S_{\max }^{\alpha}$ at different levels of $\alpha \in(0,1]$

\begin{tabular}{lllllllllll}
\hline DMU & $\alpha=0.1$ & $\alpha=0.2$ & $\alpha=0.3$ & $\alpha=0.4$ & $\alpha=0.5$ & $\alpha=0.6$ & $\alpha=0.7$ & $\alpha=0.8$ & $\alpha=0.9$ & $\alpha=1$ \\
\hline 1 & 0.33989 & 0.32143 & 0.30297 & 0.28451 & 0.26605 & 0.24759 & 0.22913 & 0.21067 & 0.19221 & 0.17375 \\
2 & 0.36731 & 0.34402 & 0.32073 & 0.29744 & 0.27415 & 0.25086 & 0.22757 & 0.20428 & 0.18099 & 0.15770 \\
3 & 0.44061 & 0.42092 & 0.40123 & 0.38154 & 0.36185 & 0.34216 & 0.32247 & 0.30278 & 0.28309 & 0.26340 \\
4 & 0.35736 & 0.33813 & 0.31889 & 0.29966 & 0.28042 & 0.26119 & 0.24195 & 0.22272 & 0.20348 & 0.18425 \\
5 & 0.59980 & 0.59085 & 0.58190 & 0.57295 & 0.56400 & 0.55505 & 0.54610 & 0.53715 & 0.52820 & 0.51925 \\
6 & 0.53659 & 0.52554 & 0.51448 & 0.50343 & 0.49237 & 0.48132 & 0.47026 & 0.45921 & 0.44815 & 0.43710 \\
7 & 0.43992 & 0.41245 & 0.38227 & 0.35750 & 0.33002 & 0.30255 & 0.27507 & 0.24760 & 0.22012 & 0.19265 \\
8 & 0.62290 & 0.60771 & 0.59252 & 0.57733 & 0.56214 & 0.54696 & 0.53177 & 0.51658 & 0.50139 & 0.48620 \\
9 & 0.53139 & 0.50003 & 0.46867 & 0.43731 & 0.40595 & 0.37459 & 0.34323 & 0.31187 & 0.28051 & 0.24915 \\
10 & 0.46541 & 0.45073 & 0.43604 & 0.42136 & 0.40667 & 0.39199 & 0.37730 & 0.36262 & 0.34793 & 0.33325 \\
11 & 0.46820 & 0.45630 & 0.44440 & 0.43250 & 0.42060 & 0.40870 & 0.39680 & 0.38490 & 0.37300 & 0.36110 \\
12 & 0.38082 & 0.36114 & 0.34146 & 0.32178 & 0.30210 & 0.28242 & 0.26274 & 0.24306 & 0.22338 & 0.20370 \\
\hline
\end{tabular}

Table 6 The values of $S_{\min }^{\alpha}$ at different levels of $\alpha \in(0,1]$

\begin{tabular}{lllllllllll}
\hline DMU & $\alpha=0.1$ & $\alpha=0.2$ & $\alpha=0.3$ & $\alpha=0.4$ & $\alpha=0.5$ & $\alpha=0.6$ & $\alpha=0.7$ & $\alpha=0.8$ & $\alpha=0.9$ & $\alpha=1$ \\
\hline 1 & 0.34639 & 0.36485 & 0.38331 & 0.40177 & 0.42023 & 0.43869 & 0.45715 & 0.47561 & 0.49407 & 0.51253 \\
2 & 0.31897 & 0.34226 & 0.36555 & 0.38884 & 0.41213 & 0.43542 & 0.45871 & 0.48200 & 0.50529 & 0.52858 \\
3 & 0.24567 & 0.26536 & 0.28505 & 0.30474 & 0.32443 & 0.34412 & 0.36381 & 0.38350 & 0.40319 & 0.42288 \\
4 & 0.32891 & 0.34815 & 0.36738 & 0.38662 & 0.40585 & 0.42509 & 0.44432 & 0.46356 & 0.48279 & 0.50203 \\
5 & 0.08648 & 0.09543 & 0.10438 & 0.11333 & 0.12228 & 0.13123 & 0.14018 & 0.14913 & 0.15808 & 0.16703 \\
6 & 0.14968 & 0.16074 & 0.17179 & 0.18285 & 0.19390 & 0.20496 & 0.21601 & 0.22707 & 0.23812 & 0.24918 \\
7 & 0.24635 & 0.27383 & 0.30130 & 0.32878 & 0.35625 & 0.38373 & 0.41120 & 0.43868 & 0.46615 & 0.49363 \\
8 & 0.06338 & 0.07857 & 0.09376 & 0.10895 & 0.12413 & 0.13932 & 0.15451 & 0.16970 & 0.18489 & 0.20008 \\
9 & 0.15489 & 0.18625 & 0.21761 & 0.24897 & 0.28033 & 0.31169 & 0.34305 & 0.37441 & 0.40577 & 0.43713 \\
10 & 0.22086 & 0.23555 & 0.25023 & 0.26492 & 0.27960 & 0.29429 & 0.30897 & 0.32366 & 0.33834 & 0.35303 \\
11 & 0.21808 & 0.22998 & 0.24188 & 0.25378 & 0.26568 & 0.27758 & 0.28948 & 0.30138 & 0.31328 & 0.32518 \\
12 & 0.30546 & 0.32514 & 0.34482 & 0.36450 & 0.38418 & 0.40386 & 0.42354 & 0.44322 & 0.46290 & 0.48258 \\
\hline
\end{tabular}


Table 7 The values of $\mathrm{RC}$ at different levels of $\alpha \in(0,1]$

\begin{tabular}{lllllllllll}
\hline DMU & $\alpha=0.1$ & $\alpha=0.2$ & $\alpha=0.3$ & $\alpha=0.4$ & $\alpha=0.5$ & $\alpha=0.6$ & $\alpha=0.7$ & $\alpha=0.8$ & $\alpha=0.9$ & $\alpha=1$ \\
\hline 1 & 0.50474 & 0.53163 & 0.55853 & 0.58543 & 0.61233 & 0.63922 & 0.66613 & 0.69303 & 0.71992 & 0.74682 \\
2 & 0.46478 & 0.49872 & 0.53265 & 0.56659 & 0.60053 & 0.63446 & 0.66840 & 0.70234 & 0.73627 & 0.77021 \\
3 & 0.35797 & 0.38666 & 0.41535 & 0.44405 & 0.47274 & 0.50143 & 0.53012 & 0.55881 & 0.58750 & 0.61619 \\
4 & 0.47926 & 0.50730 & 0.53532 & 0.56336 & 0.59138 & 0.61941 & 0.64743 & 0.67547 & 0.70349 & 0.73152 \\
5 & 0.12601 & 0.13905 & 0.15209 & 0.16514 & 0.17818 & 0.19122 & 0.20426 & 0.21730 & 0.23034 & 0.24338 \\
6 & 0.21810 & 0.23422 & 0.25032 & 0.26644 & 0.28254 & 0.29865 & 0.31475 & 0.33087 & 0.34697 & 0.36309 \\
7 & 0.35896 & 0.39899 & 0.43904 & 0.47907 & 0.51910 & 0.55914 & 0.59917 & 0.63921 & 0.67924 & 0.71928 \\
8 & 0.09235 & 0.11449 & 0.13662 & 0.15875 & 0.18087 & 0.20301 & 0.22514 & 0.24728 & 0.26941 & 0.29154 \\
9 & 0.22569 & 0.27139 & 0.31709 & 0.36278 & 0.40848 & 0.45417 & 0.49987 & 0.54556 & 0.59126 & 0.63696 \\
10 & 0.32184 & 0.34323 & 0.36462 & 0.38602 & 0.40741 & 0.42882 & 0.45021 & 0.47161 & 0.49301 & 0.51441 \\
11 & 0.31777 & 0.33511 & 0.35245 & 0.36979 & 0.38713 & 0.40447 & 0.42181 & 0.43915 & 0.45649 & 0.47383 \\
12 & 0.44509 & 0.47377 & 0.50245 & 0.53112 & 0.55980 & 0.58848 & 0.61715 & 0.64583 & 0.67451 & 0.70318
\end{tabular}

Table 8 Ranks of the DMUs at different levels of $\alpha \in(0,1]$

Ranking

$\alpha=0.1$

Existing approach [50]

Proposed approach

$\alpha=0.2$

Existing approach [50]

Proposed approach

$\alpha=0.3$

Existing approach [50]

Proposed approach

$\alpha=0.4$

Existing approach [50]

Proposed approach

$\alpha=0.5$

Existing approach [50]

Proposed approach

$\alpha=0.6$

Existing approach [50]

Proposed approach

$\alpha=0.7$

Existing approach [50]

Proposed approach

$\alpha=0.8$

Existing approach [50]

Proposed approach

$\alpha=0.9$

Existing approach [50]

Proposed approach

$\alpha=1$

Existing approach [50]

Proposed approach

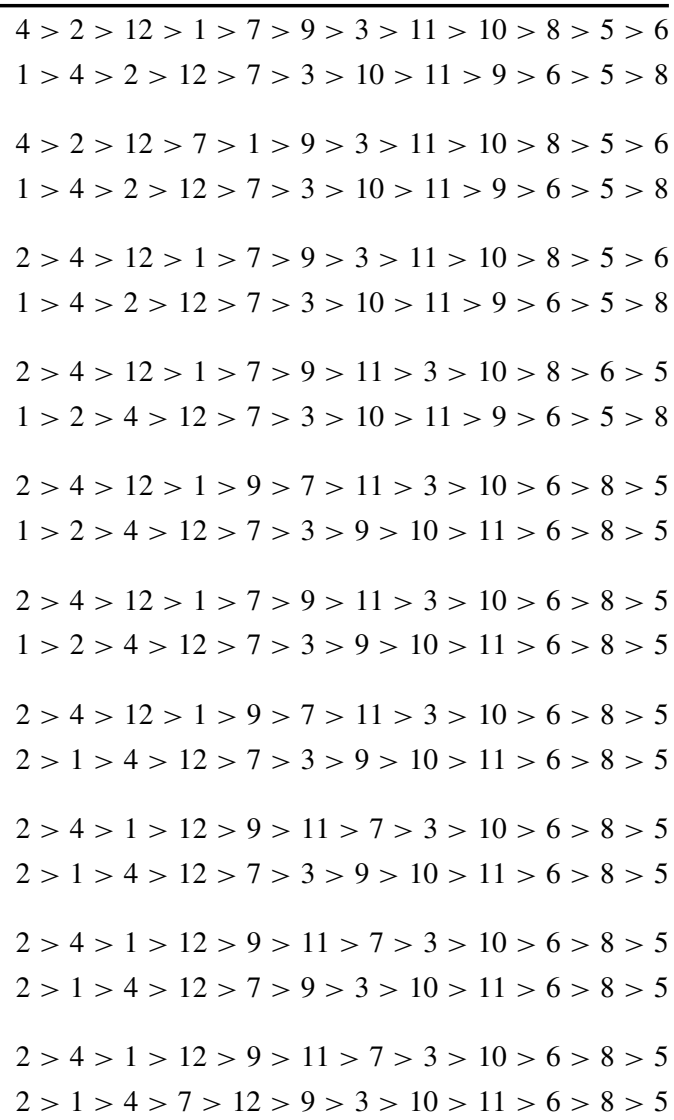




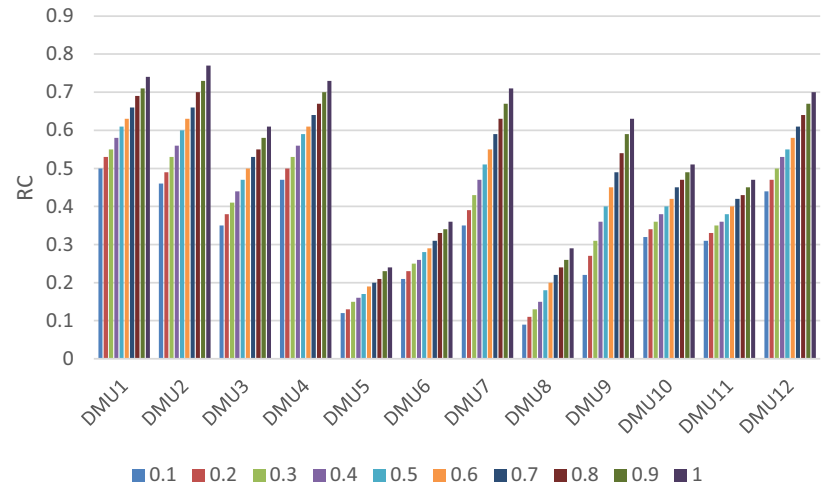

Fig. 2 The relative closeness values at different levels $\alpha \in(0,1]$

DMU ranking and can provide the decision maker with a valuable insight into the quality of management decisions and business policies.

It is worth noting that the upper bounds of the best fuzzy relative efficiency results $\left(\theta_{o}^{* U}\right)$ are same with those evaluated using crisp input-output data. The graphical representations of the efficiency results using crisp and fuzzy input-output data are shown in Fig. 5 [50] and Table 3, respectively. Carefully observing Fig. 5 and Table 3, we can analyze the impact of fuzzy data on the efficiency results of the DMUs. Table 3 reveals that $D M U_{1}, D M U_{2}$ and $D M U_{4}$ are only efficient at the upper bound of best fuzzy relative efficiency and other DMUs are not efficient, Also the upper boundary values of the best and worst fuzzy relative efficiencies of other units are less than when these are evaluated using crisp input and output data. This implies that with the variation in the data from crisp to fuzzy, the efficiency measure of almost every DMU varies. Thus, the efficiency results obtained using fuzzy input-output data are more realistic and robust as compared to the results obtained using crisp data. The complete ranking of the efficient DMUs at $\alpha \in(0,1]$, using the ranking algorithm discussed in Sect. 4.2.6, is presented in Table 8.

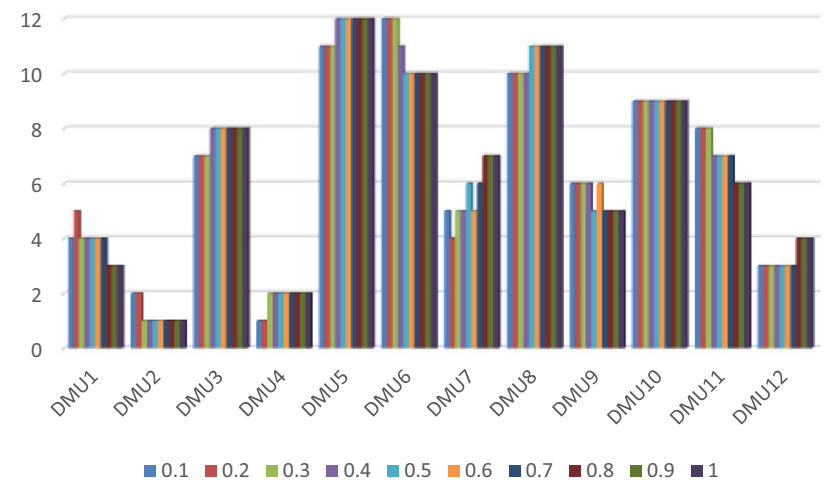

Fig. 4 Changes in DMU rankings at different levels of $\alpha \in(0,1]$ based on Puri and Yadav method [50]

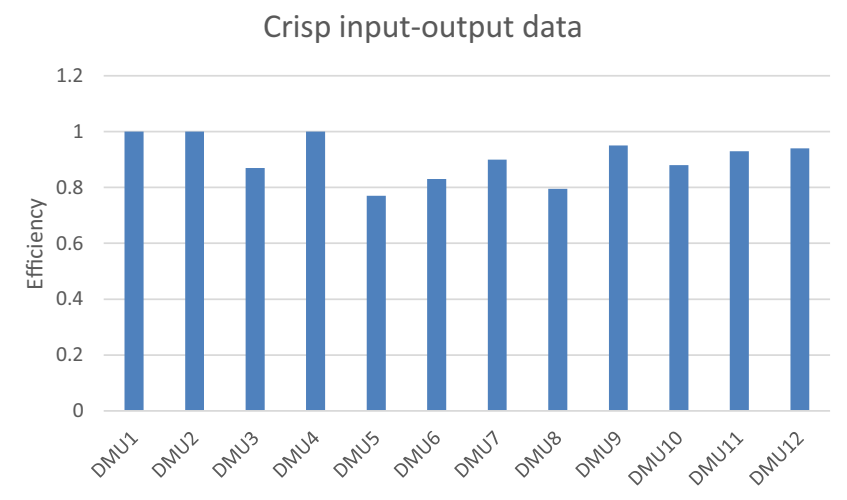

Fig. 5 Efficiency results with crisp data [50]

It shows that the ranking is different at different levels of $\alpha \in(0,1]$. Hence, we can conclude that the change at a given satisfaction level not only affects the efficiency results, but also affects the rankings of the DMUs.

To compare the ability to discriminate DMUs based on our approach with that based on Puri and Yadav's approach [50], as can be seen from Table 8, by both approaches, DMUs 11, 8, 6 and 5 have the last rank when $\alpha$ takes 0.5. However,
Fig. 3 Changes in rankings at different levels of $\alpha \in(0,1]$ based on the proposed method

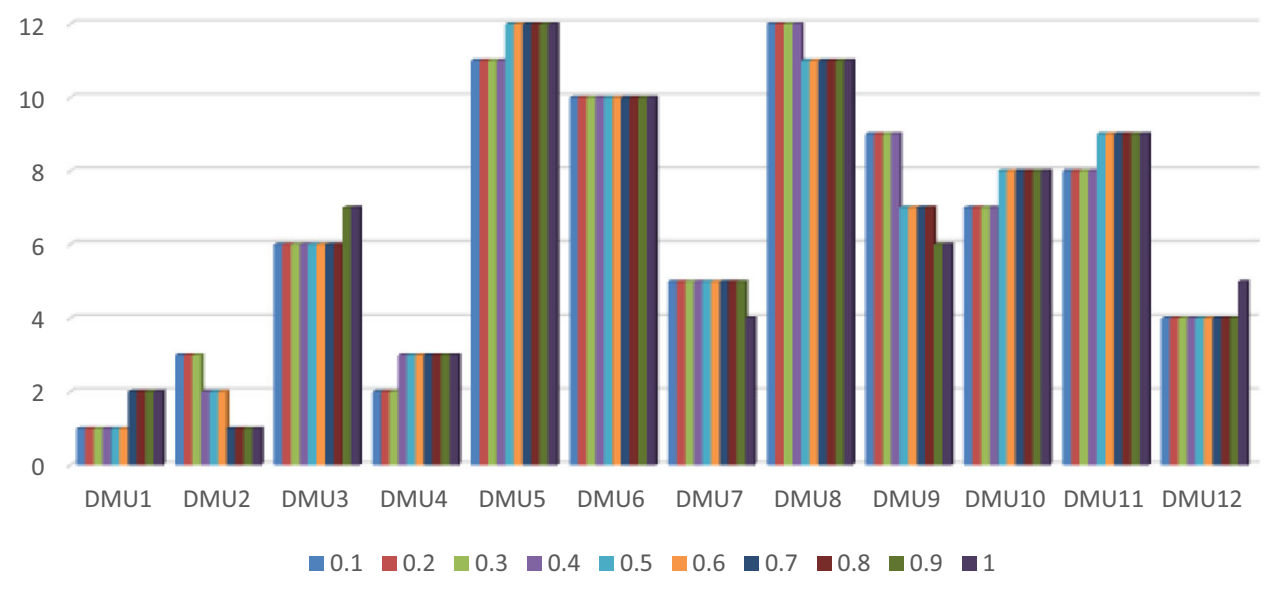


by our approach, DMUs 1, 2, 4 and 12 take the first place, second place, third place and fourth place, respectively, while by the existing approach [50], DMUs 2, 14, 12 and 1 take the first place, second place, third place and fourth place, respectively. Different from the existing approach [50] in which each DMU is evaluated based on only the optimistic perspective, each DMU is evaluated from both the optimistic and pessimistic perspectives according to our proposed approach. So, our final rank for 12 DMUS is more reasonable.

Different from the above mentioned point, the main advantages of the proposed approach over the Puri and Yadav's approach [50] to deal with undesirable outputs with fuzzy data are summarized as follows:

- The existing approach [50] only evaluates the performance of each fuzzy DMU based on the optimistic perspective, while our proposed approach provides the overall performance assessment of each fuzzy DMU from both the optimistic and pessimistic perspectives.

- Puri and Yadav [50] have used alpha-cut approach for solving fuzzy DEA models with undesirable outputs. According to this approach, the membership function is derived numerically and consequently, the membership degree of a specific efficiency must be approximated from the alpha-cut. In this study, we improved the fuzzy arithmetic approach for solving fuzzy DEA models with undesirable outputs to derive the mathematical form of the membership function of the fuzzy efficiency and consequently the membership degree can be calculated directly.

- Fuzzy efficiency measures derived from our proposed approach provide the range that all possible values derived from Puri and Yadav's approach can appear within.

- Since the efficiency measure is expressed by the membership function rather than by a crisp value, more information is provided for making decisions. This prevents the decision maker from making inappropriate decisions

- The LP problems required to solve the FDEA models based on our proposed approach have less constraints and variables compared with LP problems derived from Puri and Yadav's approach. Therefore, the main advantage of proposed method is that utilizing LP problems for solving FDEA models is highly economical compared with those derived from Puri and Yadav's approach from a computational viewpoint, regarding the number of constraints and variables.

\section{Concluding remarks}

These days a number of researchers have shown interest in the area of fuzzy data envelopment analysis and various attempts have been made to study the solution of fuzzy DEA models. In this paper we proposed a novel fuzzy DEA framework that handles fuzzy input and output data in the presence of undeniable outputs. We introduced two virtual fuzzy DMUs, namely fuzzy ideal DMU and fuzzy anti ideal DMU in the fuzzy DEA framework. Then, we measured the best fuzzy relative efficiency of FIADM and the worst fuzzy efficiency of FADMU. We used the best fuzzy relative efficiency of FIADM to find the best fuzzy relative efficiencies of DMUs and the worst fuzzy relative efficiency of FADMU to find the worst fuzzy relative efficiencies of DMUs. We combined the best and worst fuzzy efficiencies in order to find a fuzzy relative closeness of each DMU to FADMU. To provide a full ranking of performances for the DMUs, we proposed a new ranking fuzzy numbers approach based on which the DMUs were compared and ranked.

It is important to notice that traditional DEA model evaluates DMUs from the view of the best possible relative efficiency by allowing each DMU to choose the most favorable weight. According to this point of view, efficiency assessment is implemented based on the optimistic perspective which would cause the problem that all DMUs cannot be fully discriminated. To solve this problem, some scholars have proposed the common weights DEA method in order to give a complete ranking for all efficient DMUs. Also, some researchers introduced worst practice DEA models to assess the worst possible relative efficiencies for DMUs and integrated the distinctive efficiencies resulting from the optimistic and pessimistic perspectives to provide the overall performance assessment of each DMU as the basis of comparing and ranking the DMUs. In this study, we developed this approach in fuzzy environment to evaluate of DMUs with fuzzy inputs and outputs. Thus, proposing new models to extend the existing FDEA model for generating common weight in fuzzy environment is very interesting in the future research.

On the other hand, this work uses direct approaches to incorporate undesirable outputs in fuzzy DEA models. How to extend indirect approaches on modeling undesirable outputs into fuzzy DEA and analyzing their impact on the performance of fuzzy DMUs is also very interesting in the future.

Acknowledgements The authors would like to thank the anonymous reviewers and the associate editor for their insightful comments and suggestions.

\section{Compliance with ethical standards}

Conflict of Interest The authors declare that they have no conflict of interest.

Open Access This article is licensed under a Creative Commons Attribution 4.0 International License, which permits use, sharing, adaptation, distribution and reproduction in any medium or format, as long as you give appropriate credit to the original author(s) and the 
source, provide a link to the Creative Commons licence, and indicate if changes were made. The images or other third party material in this article are included in the article's Creative Commons licence, unless indicated otherwise in a credit line to the material. If material is not included in the article's Creative Commons licence and your intended use is not permitted by statutory regulation or exceeds the permitted use, you will need to obtain permission directly from the copyright holder. To view a copy of this licence, visit http://creativecomm ons.org/licenses/by/4.0/.

\section{References}

1. Amindoust A, Ahmed S, Saghafinia A (2013) Using data envelopment analysis for green supplier selection in manufacturing under vague environment. Adv Mater Res 622-623:1682-1685

2. Arana-Jiménez M, Sánchez-Gil MC, Lozano S (2020) A fuzzy DEA slacks-based approach. J Comput Appl Math. https://doi.org/ 10.1016/j.cam.2020.113180

3. Arana-Jiménez M, Sánchez-Gil MC, Lozano S (2020) Efficiency assessment and target setting using a fully fuzzy DEA approach. Int J Fuzzy Syst 22:1056-1072. https://doi.org/10.1007/s40815-0 20-00821-0

4. Arya A, Yadav SP (2019) Development of intuitionistic fuzzy data envelopment analysis models and intuitionistic fuzzy input-output targets. Soft Comput 23:8975-8993. https://doi.org/10.1007/s005 00-018-3504-3

5. Azadi M, Mirhedayatian SM, Saen RF (2013) A new fuzzy goal directed benchmarking for supplier selection. Int J Serv Oper Manag 14:321-335

6. Bagheri M, Ebrahimnejad A, Razavyan S, Hosseinzadeh Lotfi F, Malekmohammadi N (2020) Fuzzy arithmetic DEA approach for fuzzy multi-objective transportation problem. Oper Res Int J. https://doi.org/10.1007/s12351-020-00592-4

7. Banker RD (1993) Maximum likelihood, consistency and data envelopment analysis. Manag Sci 39:1265-1273

8. Banker RD, Charnes A, Cooper WW (1984) Some models for estimating technical and scale efficiencies in data envelopment analysis. Manag Sci 30:1078-1092

9. Beiranvand A, Khodabakhshi M, Yarahmadi M, Jalili M (2013) Making a mathematical programming in fuzzy systems with genetic algorithm. Life Sciences-Journal 10:50-57

10. Carlsson C, Korhonen P (1986) A parametric approach to fuzzy linear programming. Fuzzy Sets Syst 20:17-30

11. Charnes A, Cooper WW, Rhodes E (1978) Measurement the efficiency of decision making unit. Eur J Oper Res 2:429-444

12. Chen CB, Klein CM (1997) A simple approach to ranking a group of aggregated fuzzy utilities. IEEE Trans Syst Man Cybern B Cybern 27:26-35

13. Chung YH, Fare R, Grosskopf S (1997) Productivity and undesirable outputs: a directional distance function approach. J Environ Manag 51(3):229-240

14. Cooper WW, Park KS, Yu G (1999) Models for dealing with imprecise data in DEA. Manag Sci 45:597-607

15. Dubois D, Prade H (1988) Possibility theory: an approach to computerized processing of uncertainty. Plenum Press, New York

16. Ebrahimnejad A (2011) A new link between Output-oriented BCC model with fuzzy data in the present of undesirable output and MOLP. Fuzzy Inf Eng 2:113-125

17. Ebrahimnejad A, Nasseri SH, Gholami O (2019) Fuzzy stochastic data envelopment analysis with application to NATO enlargement problem. RAIRO Oper Res 53(2):705-721

18. Ebrahimnejad A, Tavana M, Nasseri SH, Gholami O (2019) A new method for solving dual DEA problems with fuzzy stochastic data. Int J Inf Technol Decis Mak 18(1):147-170
19. Ebrahimnejad A, Verdegay JL (2018) Fuzzy sets-based methods and techniques for modern analytics, volume 364 of studies in fuzziness and soft computing, 1st edn. Springer International Publishing, New York

20. Emrouznejad A, Rostamy-Malkhalifeh M, Hatami-Marbini A, Tavana M, Aghayi N (2011) An overall profit Malmquist productivity index with fuzzy and interval data. Math Comput Model 54:2827-2838

21. Färe R, Grosskopf S, Lovell CK, Pasurka C (1989) Multilateral productivity comparisons when some outputs are undesirable: a nonparametric approach. Rev Econ Stat 71(1):90-98

22. Farrel MJ (1957) The measurement of productive efficiency. J R Stat Soc 120:253-281

23. Girod O (1996) Measuring technical efficiency in a fuzzy environment. Ph.D. Dissertation, Department of Industrial and Systems Engineering, Virginia Polytechnic Institute and State University

24. Guo P, Tanaka H (2001) Fuzzy DEA: a perceptual evaluation method. Fuzzy Sets Syst 119:149-160

25. Hatami-Marbini A, Saati S (2018) Efficiency evaluation in twostage data envelopment analysis under a fuzzy environment: a common-weights approach. Appl Soft Comput 72:156-165

26. Hatami-Marbini A, Saati S (2018) An ideal-seeking fuzzy data envelopment analysis framework. Appl Soft Comput 10:1062-1070

27. Hosseinzadeh Lotfi F, Ebrahimnejad A, Vaez-Ghasemi M, Moghaddas Z (2020) Data envelopment analysis with R, volume 386 of studies in fuzziness and soft computing, 1st edn. Springer International Publishing, New York

28. Jahanshahloo GR, Hosseinzadeh Lotfi F, Shahverdi R, Adabitabar M, Rostamy- Malkhalifeh M, Sohraiee S (2009) Ranking DMUs by 11-norm with fuzzy data in DEA. Chaos Solitons Fract 39:2294-2302

29. Jahanshahloo GR, Lotfi FH, Shoja N, Tohidi G, Razavyan S (2005) Undesirable inputs and outputs in DEA models. Appl Math Comput 169(2):917-925

30. Kahraman C, Tolga E (1998) Data envelopment analysis using fuzzy concept. In: 28th international symposium on multiplevalued logic, pp 338-343

31. Kao C, Lin PH (2011) Qualitative factors in data envelopment analysis: a fuzzy number approach. Eur J Oper Res 211:586-593

32. Kao C, Liu ST (2000) Data envelopment analysis with missing data: an application to University libraries in Taiwan. J Oper Res Soc 51:897-905

33. Kao C, Liu ST (2000) Fuzzy efficiency measures in data envelopment analysis. Fuzzy Sets Syst 113:427-437

34. Kao C, Liu ST (2003) A mathematical programming approach to fuzzy efficiency ranking. Int J Prod Econ 86:45-154

35. Korhonen PJ, Luptacik M (2004) Eco-efficiency analysis of power plants: an extension of data envelopment analysis. Eur J Oper Res $154: 437-446$

36. Kwakernaak H (1978) Fuzzy random variables. I: definitions and theorems. Inf Sci 15:1-29

37. Lertworasirikul S, Fang SC, Joines JA, Nuttle HLW (2003) Fuzzy data envelopment analysis (DEA): a possibility approach. Fuzzy Sets Syst 139:379-394

38. Lertworasirikul S, Fang SC, Nuttle HLW, Joines JA (2002) Fuzzy data envelopment analysis. In: Proceedings of the 9th Bellman continuum, Beijing, p 342

39. Lertworasirikul S, Fang SC, Nuttle HLW, Joines JA (2003) Fuzzy BCC model for data envelopment analysis. Fuzzy Optim Decis Mak 2:337-358

40. Lin HT (2010) Personnel selection using analytic network process and fuzzy data envelopment analysis approaches. Comput Ind Eng 59:937-944

41. Liou TS, Wang MJ (1992) Ranking fuzzy numbers with integral value. Fuzzy Sets Syst 50:247-255 
42. Mirhedayatian M, Jelodar MJ, Adnani S, Akbarnejad M, Saen RF (2013) A new approach for prioritization in fuzzy AHP with an application for selecting the best tunnel ventilation system. Int $\mathbf{J}$ Adv Manuf Technol 68:2589-2599

43. Nasseri SH, Ebrahimnejad A, Gholami O (2018) Fuzzy stochastic data envelopment analysis with undesirable outputs and its application to banking industry. Int J Fuzzy Syst 20:534-548. https:// doi.org/10.1007/s40815-017-0367-1

44. Noura AA, Karami P (2008) Ranking functions and its application to fuzzy DEA. Int Math Forum 3:1469-1480

45. Noura AA, Natavan N, Poodineh E, Abdolalian N (2010) A new method for ranking of fuzzy decision making units by FPR/DEA Method. Appl Math Sci 4:2609-2616

46. Noura AA, Saljooghi FH (2009) Ranking decision making units in fuzzy-DEA using entropy. Appl Math Sci 3:287-295

47. Pathomsiri S, Haghani A, Dresner M, Windle RJ (2008) Impact of undesirable outputs on the productivity of US airports. Transp Res Part E Logist Transp Rev 44(2):235-259

48. Payan A, Shariff M (2013) Scrutiny Malmquist productivity index on fuzzy data by credibility theory with an application to social security organizations. J Uncertain Syst 7:36-49

49. Peykani P, Mohammadi E, Emrouznejad A, Pishvaee MS, Rostamy-Malkhalifeh M (2019) Fuzzy data envelopment analysis: an adjustable approach. Expert Syst Appl 136:439-452

50. Puri J, Yadav SP (2014) A fuzzy DEA model with undesirable fuzzy outputs and its application to the banking sector in India. Expert Syst Appl 41:6419-6432

51. Qin R, Liu Y, Liu Z-Q (2011) Modeling fuzzy data envelopment analysis by parametric programming method. Expert Syst Appl 38:8648-8663

52. Qin R, Liu Y, Liu Z, Wang G (2009) Modeling fuzzy DEA with type-2 fuzzy variable coefficients. Lecture notes in computer science. Springer, Heidelberg, pp 25-34

53. Ramezanzadeh S, Memariani A, Saati S (2005) Data envelopment analysis with fuzzy random inputs and outputs: a chanceconstrained programming approach. Iran J Fuzzy Syst 2:21-29

54. Ramík J, Rímánek JT (1985) Inequality relation between fuzzy numbers and its use in fuzzy optimization. Fuzzy Sets Syst 16:123-138

55. Razavi Hajiagha SH, Akrami H, Zavadskas EK, Hashemi SS (2013) An intuitionistic fuzzy data envelopment analysis for efficiency evaluation under uncertainty: case of a finance and credit institution. EM Econ Manag 161:128-137

56. Saati S, Hatami-Marbini A, Tavana M, Agrell PJ (2013) A fuzzy data envelopment analysis for clustering operating units with imprecise data. Int J Uncertainty Fuzziness Knowl Based Syst 21:29-54

57. Saati S, Memariani A (2005) Reducing weight flexibility in fuzzy DEA. Appl Math Comput 161:611-622

58. Saati S, Memariani A (2009) SBM model with fuzzy input-output levels in DEA. Aust J Basic Appl Sci 3:352-357

59. Saati S, Memariani A, Jahanshahloo GR (2002) Efficiency analysis and ranking of DMUs with fuzzy data. Fuzzy Optim Decis Mak $1: 255-267$

60. Scheel H (2001) Undesirable outputs in efficiency valuations. Eur J Oper Res 132(2):400-410

61. Seiford LM, Zhu J (2002) Modeling undesirable factors in efficiency evaluation. Eur J Oper Res 142(1):16-20

62. Sengupta JK (1992) A fuzzy systems approach in data envelopment analysis. Comput Math Appl 24:259-266

63. Sheth N (1999) Measuring and evaluating efficiency and effectiveness using goal programming and data envelopment analysis in a fuzzy environment. M.S. thesis, Virginia Tech., Department of Industrial and Systems Engineering, Falls Church, VA
64. Singh N, Pant M (2020) Efficiency assessment of Indian paper mills through fuzzy DEA. Mater Manuf Process 35(6):725-736. https:// doi.org/10.1080/10426914.2019.1662034

65. Soleimani-damaneh M (2009) Establishing the existence of a distance-based upper bound for a fuzzy DEA model using duality. Chaos Solitons Fract 41:485-490

66. Tanaka H, Ichihasi H, Asai K (1984) A formulation of fuzzy linear programming problem based on comparison of fuzzy numbers. Control Cybern J 13:185-194

67. Tavana M, Khanjani Shiraz R, Hatami-Marbini A, Agrell PJ, Paryab K (2013) Chance-constrained DEA models with random fuzzy inputs and outputs. Knowl Based Syst 52:32-52

68. Triantis K, Girod O (1998) A mathematical programming approach for measuring technical efficiency in a fuzzy environment. J Prod Anal 10:85-102

69. Wang YM, Greatbanks R, Yang JB (2005) Interval efficiency assessment using data envelopment analysis. Fuzzy Sets Syst 153:347-370

70. Wang YM, Luo Y (2006) DEA efficiency assessment using ideal and anti-ideal decision-making units. Appl Math Comput 173:902-915

71. Wang YM, Luo Y, Liang L (2009) Fuzzy data envelopment analysis based upon fuzzy arithmetic with an application to performance assessment of manufacturing enterprises. Expert Syst Appl 36(3):5205-5211

72. Wang YM, Yang JB, Xu DL, Chin KS (2006) On the centroids of fuzzy numbers. Fuzzy Sets Syst 157:919-926

73. Wen M, Qin Z, Kang R (2011) Sensitivity and stability analysis in fuzzy data envelopment analysis. Fuzzy Optim Decis Mak 10:1-10

74. Wu D (2006) A note on DEA efficiency assessment using ideal point: an improvement of Wang and Luo's model. Appl Math Comput 183:819-830

75. Yamashiro M (1994) The median for a trapezoidal fuzzy number. Microelectron Reliab 34:1509-1511

76. Yamashiro M (1995) The median for a L-R fuzzy number. Microelectron Reliab 35:269-271

77. Yu VF, Dat LQ (2014) An improved ranking method for fuzzy numbers with integral values. Appl Soft Comput 14:603-608

78. Zadeh LA (1965) Fuzzy sets. Inf Control 8:338-353

79. Zadeh LA (1975) The concept of a linguistic variable and its application to approximate reasoning. Inf Sci 8:199-249

80. Zadeh LA (1978) Fuzzy sets as a basis for a theory of possibility. Fuzzy Sets Syst 1:3-28

81. Zerafat AL, M., Emrouznejad, A., Mustafa, A., and Ignatius. (2013) Type-2 TOPSIS: A group decision problem when ideal values are not extreme endpoints. Group Decis Negot 22:851-866

82. Zhou SJ, Zhang ZD, Li YC (2008) Research of real estate investment risk evaluation based on fuzzy data envelopment analysis method. In: Proceedings of the international conference on risk management and engineering management, pp 444-448

Publisher's Note Springer Nature remains neutral with regard to jurisdictional claims in published maps and institutional affiliations 